\title{
AMENABILITY, FREE SUBGROUPS, AND HAAR NULL SETS IN NON-LOCALLY COMPACT GROUPS
}

\author{
SŁAWOMIR SOLECKI
}

\begin{abstract}
The paper has two objectives. On the one hand, we study left Haar null sets - a measure theoretic notion of smallness on Polish, not necessarily locally compact, groups. On the other hand, we introduce and investigate two classes of Polish groups which are closely related to this notion and to amenability. We show that left Haar null sets form a $\sigma$-ideal and have the Steinhaus property on Polish groups which are "amenable at the identity" and they lose these two properties in the presence of appropriately embedded free subgroups. As an application we prove an automatic continuity result for universally measurable homomorphisms from inverse limits of sequences of amenable, locally compact, second countable groups to second countable groups.
\end{abstract}

\section{INTRODUCTION}

All groups in this paper are assumed to be second countable. Some results can be extended to locally compact groups without a countable basis or to first countable groups, but we will not concern ourselves with these refinements.

By a Polish group we understand a topological group with a separable, metric, complete topology. All locally compact, second countable groups are Polish. However, there are many interesting Polish non-locally compact groups which can be found among the additive groups of separable Banach spaces, automorphism groups of countable structures, homeomorphism groups of compact metric spaces, countable products of locally compact, second countable groups, unitary groups of separable Hilbert spaces, isometry groups of Polish metric spaces, etc. The reader may consult [2], [11], [17], [29] for a small sample of various aspects of recent work on Polish "large", i.e., not necessarily locally compact, groups. All Polish groups are equipped with a natural notion of translation invariant, topological smallness - meager sets. If a group happens to be locally compact, a measure theoretic counterpart of meager sets is provided by Haar measure zero sets. If the group is not locally compact, left

2000 Mathematics Subject Classification. Primary: 22A10, 43A07; Secondary: 20F24, $28 \mathrm{C} 10$.

Key words and phrases. Amenable groups, free subgroups, Haar null sets.

Research supported by NSF grant DMS-0400931. 
translation invariant, locally finite, Borel measures do not exist on it. In many situations, however, there is a need for a measure theoretic notion of smallness on a general Polish group, see for example [1], [4], [7], [10], [18], [21], [23], [24], [26]. A natural and well studied such notion of smallness on all Polish groups is given by the following definition.

Definition. A subset of a Polish group $G$ is called left Haar null if there is a universally measurable set $B$ containing it and a Borel probability measure $\mu$ on $G$ such that $\mu(g B)=0$ for all $g \in G$.

Recall that a subset of a Polish group is universally measurable if it is measurable with respect to any Borel probability measure.

Left Haar null sets are well understood on locally compact, second countable groups and on Abelian Polish groups. In fact, a routine application of Fubini's theorem gives that left Haar null sets coincide with Haar measure zero sets on locally compact, second countable groups. For an introduction to left Haar null sets on Abelian Polish groups the reader may consult [4] and [8]. (Left Haar null sets are called Haar null in these references.) In the present paper, we investigate this class of measure small sets on general Polish groups.

Two test properties are asked of a family of measure small subsets of a Polish group $G$ : the family should be a proper $\sigma$-ideal (i.e., be closed under taking subsets and countable unions and not contain $G$ ) and it should fulfill the Steinhaus property (i.e., if $A$ is a universally measurable set which is not in the family, then 1 is an element of the interior of $A^{-1} A$ ). The existence of a family of subsets of a Polish group $G$ with these two properties leads immediately to an important application: each universally measurable homomorphism from $G$ to a second countable group is continuous. (In fact, the Steinhaus property was isolated in [34] as an outcome of an analysis of Frechet's and Sierpiński's work on continuity of measurable homomorphisms from $(\mathbb{R},+)$ to $(\mathbb{R},+)$. An old question of Christensen asks if any universally measurable homomorphism from a Polish group to a second countable group is continuous.)

It emerges from the present work that whether left Haar null sets have the properties discussed in the previous paragraph strongly depends on the structure of the underlying group. Two classes of groups turn out to be crucial here. Their definitions are, in a sense, localizations at the identity element of amenability and of having a discrete non-Abelian free subgroup. Positive results on left Haar null sets are obtained for groups in the first class, negative results for groups in the second class. It may be hoped that these classes of Polish groups will turn out to be important in other contexts as well.

Here is the definition of the first class. (For an explanation of the name "amenable at 1" see the remarks following the definition of amenability in Section 2.) 
Definition. A Polish group $G$ is called amenable at 1 if for any sequence $\mu_{n}, n \in$ $\mathbb{N}$, of Borel probability measures with 1 in the support of each $\mu_{n}$ there exist Borel probability measures $\nu_{n}, \nu$, for $n \in \mathbb{N}$, with $\nu_{n}$ absolutely continuous with respect to $\mu_{n}$ and such that

$$
\forall K \subseteq G \text { compact } \lim _{n} \nu * \nu_{n}(K)=\nu(K) .
$$

For a Borel probability measure $\mu$ on a Polish group $G$ let $\hat{\mu}$ be the measure defined by $\hat{\mu}(B)=\mu\left(B^{-1}\right)$ for Borel $B \subseteq G$. A quick computation shows that $\nu * \hat{\nu}_{n}\left(B^{-1}\right)=\nu_{n} * \hat{\nu}(B)$ from which it follows that in the definition of amenable at 1 group we can replace condition (1.1) by

$$
\forall K \subseteq G \text { compact } \lim _{n} \nu_{n} * \nu(K)=\nu(K)
$$

and obtain an equivalent definition.

In Subsection 3.2, we explore the extent of the class of amenable at 1 Polish groups. In Proposition 3.1, we prove that this class is closed under taking closed subgroups and continuous homomorphic images. Further, it follows from Proposition 3.2 that the following groups are amenable at 1 :

- locally compact, second countable groups;

- Abelian Polish groups;

- inverse limits of sequences of amenable, locally compact, second countable groups with continuous homomorphisms as bonding maps.

In Subsection 3.1, we prove the following theorem showing that amenability at 1 implies that left Haar null sets have the desired properties.

Theorem 1. If $G$ is an amenable at 1 Polish group, then

(i) left Haar null subsets of $G$ are closed under countable unions and, therefore, they form a proper $\sigma$-ideal;

(ii) if $A \subseteq G$ is universally measurable and not left Haar null, then $1 \in$ $\operatorname{int}\left(A^{-1} A\right)$.

A connection between Haar null sets and amenability was first hinted at in [32]. Theorem 1 generalizes results on locally compact, second countable groups [37, p.50] (see also [16, Corollary 20.17]) and on Abelian Polish groups [7], [8]. These two classical theorems were used to prove that any universally measurable homomorphism from a locally compact or an Abelian Polish group to a second countable group is continuous. The following corollary, proved in Subsection 3.1, which we deduce from the theorem above, extends both these results. Moreover, it adds, for example, countable products of amenable, locally compact, second countable groups to the class of Polish groups on which universally measurable homomorphisms are continuous.

Corollary 2. Let $G$ be an amenable at 1 Polish group. Then any universally measurable homomorphism from $G$ to a second countable group is continuous. 
In this context, one should mention two other automatic continuity results. In [6] it is proved that any universally measurable homomorphism from a Polish group to a second countable group with a left-and-right invariant metric is continuous. In [20, Theorem 5.21] Polish groups are found on which an arbitrary homomorphism to a second countable group is continuous.

In Section 4, we investigate when the properties of left Haar null sets from Theorem 1 fail to hold. The following definition provides us with an antiamenability-at- 1 property of groups which is relevant to these considerations.

Definition. A Polish group $G$ is said to have a free subgroup at 1 if it has a non-discrete free subgroup whose all finitely generated subgroups are discrete.

We will also need the following technical notion.

Definition. A Polish group is strongly non-locally compact if for any neighborhood $U$ of 1 there exists a neighborhood $V$ of 1 such that $U$ cannot be covered by finitely many sets of the form $g V h$ with $g, h \in G$.

In Subsections 4.3.1 and 4.3.2, we derive various criteria for a Polish group to have a free subgroup at 1 and to be strongly non-locally compact the most interesting of which, Proposition 4.4, gives a sufficient condition for strong non-local compactness in terms of distal-type actions of the group. We apply these criteria to show that the well studied Polish groups on the following list fulfill both these conditions:

- countable products of Polish groups containing discrete free non-Abelian subgroups;

- $S_{\infty}$ the group of all permutations of a countably infinite set;

- the group of all permutations of $\mathbb{Q}$ which preserve the standard linear order;

- the group of all homeomorphisms of $2^{\mathbb{N}}$;

- the group of all measure preserving homeomorphisms of $2^{\mathbb{N}}$ where $2^{\mathbb{N}}=$ $\{0,1\}^{\mathbb{N}}$ is equipped with the Lebesgue measure which is the product of measures on $\{0,1\}$ assigning weight $1 / 2$ to 0 and to 1 ;

- the groups of all homeomorphisms of $[0,1]^{n}, n \in \mathbb{N}$;

- the completion of the free group on $\mathbb{N}^{\mathbb{N}}$ with the Graev metric [12]; this group is a surjectively universal group for Polish groups with two-sided invariant metrics.

The following theorem proved in Subsection 4.1 shows that both properties: of being a $\sigma$-ideal and of having the Steinhaus property break down for left Haar null sets in the presence of a free subgroup at 1 . In relation to point (i) below notice that left Haar null sets are invariant under right (and, of course, left) translations.

Theorem 3. Let $G$ be a Polish group. Assume $G$ has a free subgroup at 1 . 
(i) There exists a universally measurable (even Borel) set $B \subseteq G$ such that $B$ is left Haar null and $B \cup B g=G$ for some $g \in G$.

(ii) Assume, additionally, that $G$ is strongly non-locally compact. Then there exists a universally measurable (even Borel) set $A \subseteq G$ which is not left Haar null and $1 \notin \operatorname{int}\left(A^{-1} A\right)$ holds.

An interesting feature of the construction in point (ii) above are certain estimates derived from isoperimetric inequalities on free groups. We hope this construction will find other applications.

Note that Theorems 1(i) and 3(i) imply that amenability at 1 and having a free subgroup at 1 exclude each other. There do, however, exist Polish groups with a free subgroup at 1 which are extremely amenable, that is, all their continuous actions on compact spaces have fixed points, see $\S 4.3 .3$, Example 2.

The next corollary, which is an immediate consequence of Theorem 3, indicates that a new approach may be needed to prove continuity of universally measurable homomorphisms defined on groups as in the assumptions of Theorem 3 , if such a result is true at all.

Corollary 4. Let $G$ be a Polish group containing a free subgroup at 1 and being strongly non-locally compact. There is no family $\mathcal{F}$ of subsets of $G$ which has the following three properties:

(i) $\mathcal{F}$ contains all left Haar null sets or is contained in the family of left Haar null sets;

(ii) $\mathcal{F}$ is closed under taking finite unions and $G \notin \mathcal{F}$;

(iii) 1 is in the interior of $A^{-1} A$ for any Borel set $A \subseteq G$ with $A \notin \mathcal{F}$.

As a by-product of the method used to prove Theorem 3, in Subsection 4.2 we show the following result which may be of independent interest.

Theorem 5. Let $G$ be a locally compact, second countable group. There exists a neighborhood $V$ of 1 such that no two elements of $V$ generate a discrete, nonAbelian, free subgroup of $G$.

There exists another well studied notion of measure theoretic smallness on Polish groups - Haar null sets. Below we compare this notion with left Haar null sets and indicate some consequences for Haar null sets that can be derived from Theorem 3.

Definition. A subset of a Polish group $G$ is called Haar null if it is included in a universally measurable set $B$ such that for some Borel probability measure $\mu$ on $G$ we have $\mu(g B h)=0$ for all $g, h \in G$.

If the group $G$ is locally compact, Haar null sets coincide with Haar measure zero sets [7], [8], [35]. Obviously, Haar null sets are left Haar null and these 
families coincide on locally compact, second countable and on Abelian Polish groups. As proved in [30] in general the two notions are not identical.

Haar null sets form a $\sigma$-ideal on an arbitrary Polish group [7], [8], [35]. The status of the Steinhaus property for this family is, however, very different. It does hold on locally compact, second countable and on Abelian Polish groups, [37, p.50], [7]. Notice, however, that on these types of groups Haar null sets coincide with left Haar null sets. It was shown in [33] that Haar null sets lose the Steinhaus property on some countable products of countable discrete groups even if the algebraic structure of the factors is not far from Abelian. Moreover, this happens on such product groups precisely when Haar null sets cease to be equal to left Haar null sets. Here is the precise statement from [33].

Let $H_{n}, n \in \mathbb{N}$, be countable discrete groups. The following conditions are equivalent.

(i) If $A \subseteq \prod_{n} H_{n}$ is a universally measurable set which is not Haar null, then 1 is in the interior of $A^{-1} A$.

(ii) Left Haar null and Haar null sets coincide on $\prod_{n} H_{n}$.

(iii) For all but finitely many $n$, all elements of $H_{n}$ have finite conjugacy classes.

Recall that finite conjugacy class groups form a small subclass of amenable groups. Therefore, the above quoted theorem and the remarks preceding it and the main results of the present work suggest that, at least when the two test questions considered in this paper are concerned, left Haar null sets seem to be a more appropriate notion of measure small sets than Haar null sets.

Be it as it may, Theorem 3 can also be used to obtain interesting consequences for Haar null sets. Mycielski [24] inquired if Haar null sets are always equal to left Haar null sets and Christensen [8] and Mycielski [24] asked if the Steinhaus property holds for Haar null sets. It was shown in [30] that on the group of all increasing homeomorphisms of $[0,1]$ the first question has a negative answer. The result from [33] quoted above provides examples of groups answering both questions in the negative. All these results give examples of rather particular natures. The corollary to Theorem 3 stated below and proved in Subsection 4.2 shows that both questions above have negative answers on a large class of Polish groups (see Subsections 4.3.1 and 4.3.2).

Corollary 6. Let $G$ be a Polish group with a free subgroup at 1 .

(i) Haar null subsets of $G$ form a proper subfamily of left Haar null sets.

(ii) If $G$ is additionally strongly non-locally compact, then there exists a Borel non-Haar null subset $A$ of $G$ with 1 not in the interior of $A^{-1} A$.

We finish the paper with stating some open questions in Section 5. 


\section{Definitions And nOtation}

If $X$ is a Polish space, let $\mathcal{K}(X)$ be the space of all compact subsets of $X$ with the Vietoris topology. It is a Polish space. Moreover, a metric $d$ on $X$ induces the Hausdorff metric on $\mathcal{K}(X)$, which is compatible with the Vietoris topology, as follows: $\emptyset$ is at distance 1 from any nonempty set in $\mathcal{K}(X)$ and the distance between two nonempty $K, L \in \mathcal{K}(X)$ is given by the formula

$$
\max \left(\sup _{x \in K} \inf _{y \in L} d(x, y), \sup _{y \in L} \inf _{x \in K} d(x, y)\right) .
$$

We denote the Hausdorff metric again by $d$.

We will also need the space $P(X)$ of all probability Borel measures on $X$ with the topology induced by the functions $P(X) \ni \mu \rightarrow \int_{X} f d \mu \in \mathbb{R}$ for continuous bounded $f: X \rightarrow \mathbb{R}$. This is also a Polish topology. The set $P(X)$ is a subset of the normed linear space consisting of all signed countably additive measures on $X$. The norm we consider this space to be equipped with is $\|\nu\|=\sup \{|\nu(B)|: B \subseteq X$ Borel $\}[9$, p. 86]. The topology this norm induces on $P(X)$ is stronger than the Polish topology described above. For $x \in X$ let $\delta_{x}$ stand for the measure given by $\delta_{x}(A)=1$ if $x \in A$ and $\delta_{x}(A)=0$ if $x \notin A$. For $\mu \in P(X)$, let the support of $\mu$, in symbols $\operatorname{supp}(\mu)$, be the set

$$
X \backslash \bigcup\{U: U \text { is open and } \mu(U)=0\} .
$$

We write $\nu \ll \mu$ if $\nu$ is absolutely continuous with respect to $\mu$, that is, if all Borel (or, equivalently, universally measurable) $\mu$-measure zero sets are $\nu$ measure zero. If $\pi: X \rightarrow Y$ is a Borel function, $X$ and $Y$ are Polish spaces, and $\mu$ is a Borel measure on $X$ let $\pi_{*} \mu$ be a Borel measure on $Y$ given by

$$
\left(\pi_{*} \mu\right)(B)=\mu\left(\pi^{-1}(B)\right)
$$

for a Borel set $B \subseteq Y$.

Let $G$ be a Polish group. For two measures $\mu, \nu \in P(G)$ recall that the convolution $\mu * \nu$ is defined by letting

$$
\int_{G} f d(\mu * \nu)=\int_{G} \int_{G} f(x y) d \mu(x) d \nu(y)
$$

for a bounded Borel function $f: G \rightarrow \mathbb{R}$. If $f: G \rightarrow \mathbb{R}$ is a Borel function and $g \in G$, let

$$
{ }_{g} f(x)=f\left(g^{-1} x\right) \text { and } f_{g}(x)=f\left(x g^{-1}\right) .
$$

If $\mu \in P(G)$ and $g \in G$, define measures ${ }_{g} \mu, \mu_{g} \in P(G)$ by letting

$$
\int_{G} f d\left(g_{g} \mu\right)=\int_{G}\left(g^{-1} f\right) d \mu \text { and } \int_{G} f d\left(\mu_{g}\right)=\int_{G}\left(f_{g^{-1}}\right) d \mu
$$

for a bounded Borel function $f: G \rightarrow \mathbb{R}$. So for a Borel set $B \subseteq G$, we have ${ }_{g} \mu(B)=\mu\left(g^{-1} B\right)$ and $\mu_{g}(B)=\mu\left(B g^{-1}\right)$. 
Recall that a locally compact group $H$ is amenable if there exists a linear functional $m$ from the linear space $C_{b}(G)$ of all real valued, bounded, continuous functions from $G$ to $\mathbb{R}$ such that

(i) $m\left({ }_{g} f\right)=m(f)$ for any $f \in C_{b}(G)$ and any $g \in G$ and

(ii) $m(f) \geq 0$ for any non-negative $f \in C_{b}(G)$ and $m(\mathbf{1})=1$ where $\mathbf{1}$ is the function constantly equal to 1 .

There exists a large variety of conditions characterizing amenability of locally compact groups. Our definition of amenability at 1 is not analogous to a standard such condition. However, it is not difficult to show that the following condition on a locally compact, second countable group $G$ is equivalent to the property in [27, Theorem 4.4(iii) for $p=1]$ which, in turn, is equivalent to amenability.

For any Borel probability measure $\mu$ on $G$ and any $\epsilon>0$ there exists a Borel probability measure $\nu$ such that for any compact set $K \subseteq G$ we have $\mid \nu *$ $\mu(K)-\nu(K) \mid<\epsilon$

This condition makes the analogy between amenability at 1 and amenability clearer.

Let $\mathbb{N}$ stand for the set of all natural numbers including 0 . By 2 we denote the set $\{0,1\}$. So, for example, $2^{\mathbb{N}}$ is the compact metric space $\{0,1\}^{\mathbb{N}}$ where $\{0,1\}$ is taken with the discrete topology.

If $A \subseteq X \times Y$ for some sets $X, Y$, for $x_{0} \in X$ and $y_{0} \in Y$ let

$$
A_{x_{0}}=\left\{y \in Y:\left(x_{0}, y\right)\right\} \text { and } A^{y_{0}}=\left\{x \in X:\left(x, y_{0}\right)\right\}
$$

\section{Amenability at 1 And left HaAR null Sets}

\subsection{Left Haar null sets and automatic continuity on amenable at 1}

groups. In the present subsection we establish Theorem 1 and Corollary 2.

Proof of Theorem 1. (i) Let $A_{n} \subseteq G, n \in \mathbb{N}$, be left Haar null. We can assume that each $A_{n}$ is universally measurable. Note that if a Borel probability measure witnesses that a set is left Haar null, then so does any left translate of the measure. This observation allows us to pick a sequence $\left(\mu_{k}\right)$ of Borel probability measures such that for any $n$ there are infinitely many $k$ with $\mu_{k}$ witnessing that $A_{n}$ is left Haar null and with $1 \in \operatorname{supp}\left(\mu_{k}\right)$. Since $G$ is amenable at 1 , we can find $\nu_{k}, \nu \in P(G)$ such that $\nu_{k} \ll \mu_{k}$ and for any compact $K$,

$$
\lim _{k} \nu * \nu_{k}(K)=\nu(K) .
$$


Note that, since $\nu_{k} \ll \mu_{k}$, for a given $n$ there is infinitely many $k$ such that for all $h \in G$ we have $\nu_{k}(h A)=0$. Therefore, for any $g \in G$

$$
\liminf _{k} \nu * \nu_{k}\left(g A_{n}\right)=\liminf _{k} \int_{G} \nu_{k}\left(x^{-1} g A_{n}\right) d \nu(x)=0 .
$$

Thus, the same is true for any compact subset $K$ of $A_{n}$, hence by $(3.1) \nu(g K)=$ 0 for any such compact set. It follows from it that, since $A_{n}$ is universally measurable, $\nu\left(g A_{n}\right)=0$. Since this holds for each $n, \nu\left(g\left(\bigcup_{n} A_{n}\right)\right)=0$ for any $g \in G$, which implies that $\bigcup_{n} A_{n}$ is left Haar null.

(ii) Let $A \subseteq G$ be a universally measurable set which is not left Haar null. Assume towards contradiction that 1 is not in the interior of $A^{-1} A$. This means that we can pick a sequence $g_{n} \rightarrow 1$ as $n \rightarrow \infty$ with

$$
g_{n} \notin A^{-1} A \text {. }
$$

Consider the sequence of measures $\mu_{k}=\sum_{n \geq k} 2^{k-n-1} \delta_{g_{n}}, k \in \mathbb{N}$, and note that $1 \in \operatorname{supp}\left(\mu_{k}\right)$ for each $k$. Applying to it the definition of amenability at 1 , we get Borel probability measures $\nu_{k} \ll \mu_{k}$ and $\nu$ such that for any compact set $K$ we have

$$
\lim _{k} \nu * \nu_{k}(K)=\nu(K) .
$$

We claim that $\nu$ witnesses that $A$ is left Haar null. Assume the opposite, that is, $\nu\left(h_{0} A\right)>0$ for some $h_{0} \in G$. Note first that since $\nu_{k} \ll$ $\sum_{n \geq k} 2^{k-n-1} \delta_{g_{n}}$, we have $\nu_{k}=\sum_{n \geq k} \alpha_{n, k} \delta_{g_{n}}$ with $\alpha_{n, k} \geq 0$ and $\sum_{n \geq k} \alpha_{n, k}=$ 1 and, therefore, for any compact set $K$

$$
\nu * \nu_{k}(K)=\sum_{n \geq k} \alpha_{n, k} \nu\left(K g_{n}^{-1}\right) .
$$

Fix now an open set $U_{0} \supseteq A$ with

$$
\nu\left(h_{0} U_{0} \backslash h_{0} A\right)<\frac{1}{2} \nu\left(h_{0} A\right) .
$$

Pick a compact subset $K_{0}$ of $A$ so that $\nu\left(h_{0} K_{0}\right)>(1 / 2) \nu\left(h_{0} A\right)$. Combining this with (3.3) and (3.4) applied to $K=h_{0} K_{0}$, we see that for large enough $k$,

$$
\sum_{n \geq k} \alpha_{n, k} \nu\left(h_{0} K_{0} g_{n}^{-1}\right)>\frac{1}{2} \nu\left(h_{0} A\right) .
$$

Since the numbers $\alpha_{n, k}$ are non-negative and add up to 1 , it follows from the last formula that there exists $n_{k} \geq k$ such that

$$
\mu\left(h_{0} K_{0} g_{n_{k}}^{-1}\right)>\frac{1}{2} \mu\left(h_{0} A\right) .
$$

Since $g_{n_{k}} \rightarrow 1$ as $k \rightarrow \infty$, for large enough $k$ we also have $h_{0} K_{0} g_{n_{k}}^{-1} \subseteq h_{0} U_{0}$. Thus, in the light of (3.5) and (3.6), we have an $n$ such that

$$
h_{0} K_{0} g_{n}^{-1} \cap h_{0} A \neq \emptyset \text {. }
$$


This gives $g_{n} \in A^{-1} K_{0}$ which is a direct contradiction with (3.2) since $K_{0} \subseteq$ $A$.

Proof of Corollary 2. This is a routine argument from Theorem 1. Let $f$ : $G \rightarrow H$ be a universally measurable homomorphism with $G$ amenable at 1 and $H$ second countable. It suffices to show that $f$ is continuous at 1 . We can assume that $f$ is surjective. Let $W$ be an open neighborhood of 1 in $H$. Let $V$ be an open neighborhood of 1 in $H$ with $V^{-1} V \subseteq W$. Then countably many translates of $V$ cover $H$. Since $f$ is onto, countably many translates of $f^{-1}(V)$ cover $G$. Thus, $f^{-1}(V)$ is not Haar null by Theorem 1(i). Since it is also universally measurable, by Theorem 1(ii), 1 is in the interior of

$$
f^{-1}(V)^{-1} f^{-1}(V)=f^{-1}\left(V^{-1} V\right) \subseteq f^{-1}(W) .
$$

Since $W$ was an arbitrary open neighborhood of 1 , we are done.

3.2. Extent of the class of amenable at 1 groups. Let $X$ be a Polish space. A simple argument shows that if $\mu_{n}, \mu \in P(X), n \in \mathbb{N}$, and $\liminf _{n} \mu_{n}(K) \geq \mu(K)$ for all compact $K \subseteq X$, then for any bounded Borel function $f: X \rightarrow \mathbb{R}$, we have $\lim _{n} \int_{X} f d \mu_{n}=\int_{X} f d \mu$. This last condition, in turn, is equivalent by Nikodym's Theorem [9, p. 90] to weak convergence of $\left(\mu_{n}\right)$ to $\mu$. Here we consider $P(X)$ as a subset of the normed linear space of all signed Borel countably additive measures on $X$ (see Section 2). Thus, we have the following simple lemma.

Lemma 3.1. Let $G$ be a Polish group. The following conditions are equivalent

(i) $G$ is amenable at 1 ;

(ii) for any sequence $\mu_{n} \in P(G), n \in \mathbb{N}$, with $1 \in \operatorname{supp}\left(\mu_{n}\right)$ there exist $\nu_{n}, \nu \in P(G)$ such that $\nu_{n} \ll \mu_{n}$ and $\liminf _{n} \nu * \nu_{n}(K) \geq \nu(K)$ for any $K \subseteq G$ compact;

(iii) for any sequence $\mu_{n} \in P(G), n \in \mathbb{N}$, with $1 \in \operatorname{supp}\left(\mu_{n}\right)$ there exist $\nu_{n}, \nu \in P(G)$ such that $\nu_{n} \ll \mu_{n}$ and for any bounded Borel function $f: G \rightarrow \mathbb{R}$

$$
\lim _{n} \int_{G} f d\left(\nu * \nu_{n}\right)=\int_{G} f d \nu
$$

(iv) for any sequence $\mu_{n} \in P(G), n \in \mathbb{N}$, with $1 \in \operatorname{supp}\left(\mu_{n}\right)$ there exist $\nu_{n}, \nu \in P(G)$ such that $\nu_{n} \ll \mu_{n}$ and the sequence $\left(\nu * \nu_{n}\right)$ weakly converges to $\nu$.

Proposition 3.1. A closed subgroup of an amenable at 1 Polish group is amenable at 1. A Polish group which is a continuous homomorphic image of an amenable at 1 Polish group is itself amenable at 1.

Proof. We deal with closed subgroups first. Let $G$ be amenable at 1 and let $H<G$ be a closed subgroup. Let $\mu_{n}$ be Borel probability measures on $H$ 
with $1 \in \operatorname{supp}\left(\mu_{n}\right)$. These are also measures on $G$ so there exist $\nu_{n} \ll \mu_{n}$ and $\nu$ in $P(G)$ with $\lim _{n} \nu * \nu_{n}(B)=\nu(B)$ for any Borel $B \subseteq G$. Of course, the supports of the measures $\nu_{n}$ are included in $H$ so, to show that $H$ is amenable at 1 , it will suffice to produce a Borel probability measure $\nu^{\prime}$ with the support included in $H$ so that $\lim _{n} \nu^{\prime} * \nu_{n}(B)=\nu^{\prime}(B)$ for any Borel $B \subseteq H$. Let $S \subseteq G$ be a Borel set which selects precisely one point from each left coset of $H$, see [19, Theorem 12.17]. Define $\nu^{\prime}(B)=\nu(S B)$ for $B \subseteq H$ Borel. Note that by [19, Theorem 15.1] $S B$ is a Borel subset of $G$, and it is easy to check that this formula defines a Borel probability measure on $H$. Now note that for a Borel set $B \subseteq H$ we have

$$
\nu^{\prime} * \nu_{n}(B)=\int_{H} \nu\left(S B y^{-1}\right) d \nu_{n}(y)=\int_{G} \nu\left(S B y^{-1}\right) d \nu_{n}(y)=\nu * \nu_{n}(S B),
$$

where the second equality holds because the support of $\nu_{n}$ is included in $H$. Thus,

$$
\lim _{n} \nu^{\prime} * \nu_{n}(B)=\lim _{n} \nu * \nu_{n}(S B)=\nu(S B)=\nu^{\prime}(B) .
$$

We show now that Polish, amenable at 1 groups are closed under taking continuous homomorphic images. To this end it will suffice to consider a Polish group $G$ which is amenable at 1 and a closed normal subgroup $H \triangleleft G$ and show that $G / H$ is amenable at 1 . Let $\pi: G \rightarrow G / H$ be the quotient map. For any Borel probability measure $\rho$ on $G / H$ with 1 in its support we can find a Borel probability measure $\tilde{\rho}$ on $G$ with 1 in its support such that $\pi_{*}(\tilde{\rho})=\rho$. This is arranged as follows. Fix a descending sequence $U_{n}$, $n \in \mathbb{N}$, of open neighborhoods of 1 in $G$ forming a basis at 1 with $U_{0}=G$. Let $S_{n} \subseteq U_{n}$ be a Borel set which selects one point from each coset $g H$ of $H$ such that $g H \cap U_{n} \neq \emptyset$ and $g H \cap U_{n+1}=\emptyset$, see [19, Theorem 12.16]. Let $S=\{1\} \cup \bigcup_{n} S_{n}$. Then $S$ is a Borel subset of $G$ such that $\pi \uparrow S: S \rightarrow G / H$ is a bijection and for any open $U \ni 1,(\pi \uparrow S)(U)$ is a neighborhood of 1 in $G / H$. Given $\rho$ on $G / H$ let $\tilde{\rho}=\left((\pi \uparrow S)^{-1}\right)_{*}(\rho)$. One easily checks that it is as required.

Let $\mu_{n}$ be Borel probability measures on $G / H$ with $1 \in \operatorname{supp}\left(\mu_{n}\right)$. Define $\tilde{\mu}_{n}$ on $G$ as in the paragraph above, that is, so that $1 \in \operatorname{supp}\left(\tilde{\mu}_{n}\right)$ and $\pi_{*}\left(\tilde{\mu}_{n}\right)=\mu_{n}$. Since $G$ is amenable at 1 , there exist Borel probability measures $\tilde{\nu}_{n} \ll \tilde{\mu}_{n}$ and $\tilde{\nu}$ on $G$ such that for any Borel set $B \subseteq G$,

$$
\lim _{n} \tilde{\nu} * \tilde{\nu}_{n}(B)=\tilde{\nu}(B) .
$$

Define $\nu_{n}=\pi_{*}\left(\tilde{\nu}_{n}\right)$ and $\nu=\pi_{*}(\tilde{\nu})$. Clearly $\nu_{n} \ll \mu_{n}$. For a Borel set $B \subseteq G / H$ and $n \in \mathbb{N}$, the Borel function $G \ni g \rightarrow \tilde{\nu}_{n}\left(g \pi^{-1}(B)\right) \in \mathbb{R}$ is constant on cosets of $H$ and, therefore,

$$
\int_{G} \tilde{\nu}_{n}\left(g \pi^{-1}(B)\right) d \tilde{\nu}(g)=\int_{G / H} \tilde{\nu}_{n}\left(\pi^{-1}(x B)\right) d \nu(x) .
$$


Using this identity one shows by a simple calculation that

$$
\nu * \nu_{n}(B)=\tilde{\nu} * \tilde{\nu}_{n}\left(\pi^{-1}(B)\right)
$$

Now it follows from this, $\nu(B)=\tilde{\nu}\left(\pi^{-1}(B)\right)$, and (3.7) that $G / H$ is amenable at 1 .

Proposition 3.2. The class of Polish groups which are amenable at 1 contains

(i) Abelian Polish groups;

(ii) products $\prod_{n} H_{n}$ where each $H_{n}$ is locally compact, second countable and all but finitely many of them are amenable.

(iii) locally compact, second countable groups and inverse limits of sequences of amenable, locally compact, second countable groups with continuous homomorphisms as bonding maps.

Proof. Notice that (iii) follows from (ii) and Proposition 3.1. Indeed, if $G$ is locally compact, second countable, then $G$ can be identified with the product of itself and countably many copies of the trivial (amenable) group consisting of the identity only. Since inverse limits of sequences of amenable, locally compact, second countable groups with continuous homomorphisms as bonding maps are closed subgroups of products as in point (ii) above, it follows from Proposition 3.1 that such inverse limits are amenable at 1.

Let $G$ be an Abelian Polish group. For $\mu \in P(G)$ and $n \in \mathbb{N}$ we write $\mu^{n}$ for $\mu * \cdots * \mu$ where the convolution is taken $n$ times with $\mu^{0}$ equal to $\delta_{1}$. Let $\mu_{k} \in P(G), k \in \mathbb{N}$, contain 1 in their supports. Fix a translation invariant metric $d$ on $G$. Since the group is Polish and Abelian, $d$ is complete, see [17, Lemma 7.4]. Let $\nu_{k}$ be the measure obtained by normalizing the restriction of $\mu_{k}$ to the ball around 1 of radius $1 /\left((k+1) 2^{k}\right)$. Define measures $\rho_{k}$ by

$$
\rho_{k}=\frac{1}{k+1} \sum_{i=0}^{k} \nu_{k}^{i} .
$$

(There are several ways of defining $\rho_{k}$ to make the rest of the argument work. It was originally set to be $\left(\frac{1}{2} \delta_{1}+\frac{1}{2} \nu_{k}\right)^{n_{k}}$ for large enough $n_{k} \in \mathbb{N}$. The simpler formula above was suggested by Robert Kaufman.) Note first that since $d$ is translation invariant, the support of $\rho_{k}$ is included in a ball around 1 of radius $2^{-k}$. Furthermore, for any Borel function $f: G \rightarrow[0,1]$, we have

$$
\begin{aligned}
(k+1)\left|\int_{G} f d \rho_{k}-\int_{G} f d\left(\rho_{k} * \nu_{k}\right)\right| & =\left|\sum_{i=0}^{k} \int_{G} f d \nu_{k}^{i}-\sum_{i=0}^{k} \int_{G} f d\left(\nu_{k}^{i} * \nu_{k}\right)\right| \\
& =\left|f(1)-\int_{G} f d \nu_{k}^{k+1}\right| \leq 1,
\end{aligned}
$$


from which we get

$$
\left|\int_{G} f d \rho_{k}-\int_{G} f d\left(\rho_{k} * \nu_{k}\right)\right| \leq \frac{1}{k+1} .
$$

Define now

$$
\nu=*_{k} \rho_{k} .
$$

Since the support of $\rho_{k}$ is included in a ball around 1 of radius $2^{-k}$ and since $d$ is complete and translation invariant, the infinite convolution in the definition of $\nu$ converges. Let $K \subseteq G$ be compact. Then since $G$ is Abelian, using (3.8) we obtain for each $k$

$$
\begin{aligned}
\left|\nu(K)-\nu * \nu_{k}(K)\right| & =\left|\nu(K)-\int_{G}\left(*_{i \neq k} \rho_{i}\right)\left(x^{-1} K\right) d\left(\rho_{k} * \nu_{k}\right)(x)\right| \\
& \leq\left|\nu(K)-\int_{G}\left(*_{i \neq k} \rho_{i}\right)\left(x^{-1} K\right) d \rho_{k}(x)\right|+\frac{1}{k+1} \\
& =\frac{1}{k+1},
\end{aligned}
$$

hence $\lim _{k} \nu * \nu_{k}(K)=\nu(K)$ and point (i) follows.

(ii) We can assume without loss of generality that all $H_{n}$ with $n>0$ are amenable. For a Haar positive set $C$, we write $\lambda_{C}$ for the normalized restriction to $C$ of a right invariant Haar measure. Let $\pi_{n}$ be the projection from $\prod_{i} H_{i}$ on $H_{n}$ and let $\sigma_{n}$ be the projection on $\prod_{i \leq n} H_{i}$. Given $i \in \mathbb{N}$ and a Haar positive compact set $C \subseteq H_{i}$, let $P_{i, C}(h, k)$, with $h \in H_{i}$ and $k \in \mathbb{N}$, stand for the formula

$$
\forall f \quad \int_{H_{i}} f(x h) d \lambda_{C}(x)>\int_{H_{i}} f(x) d \lambda_{C}(x)-2^{-i-k}
$$

where $f$ ranges over Borel functions $f: H_{i} \rightarrow[0,1]$.

Let $\mu_{k}, k \in \mathbb{N}$, be Borel probability measures on $\prod_{n} H_{n}$ with $1 \in \operatorname{supp}\left(\mu_{k}\right)$ for all $k$. Find first Borel probability measures $\nu_{k}^{\prime} \ll \mu_{k}$ so that $\operatorname{supp}\left(\nu_{k}^{\prime}\right)$ is compact and contains 1 . For each $i$, we can find a compact Haar positive set $C_{i} \subseteq H_{i}$ such that $P_{i, C_{i}}(h, k)$ holds for all $k<i$ and all $h \in \pi_{i}\left(\operatorname{supp}\left(\nu_{k}^{\prime}\right)\right)[27$, Theorem 4.10]. This is possible since for each $i>0, H_{i}$ is amenable. Notice that since there are no $k \in \mathbb{N}$ with $k<0$, for $i=0$ any compact Haar positive set $C_{0}$ will do.

Restrict $\nu_{k}^{\prime}$ to so small a neighborhood $V$ of 1 that $P_{i, C_{i}}(h, k)$ holds for any $i \leq k$ and any $h \in \pi_{i}(V)$. This is possible by the following argument. If $\left(V_{p}\right)_{p \in \mathbb{N}}$ is an open neighborhood basis at 1 in $H_{i}$ with $V_{p+1} \subseteq V_{p}$ for each $p$, $C_{i}=\bigcap_{p} C_{i} V_{p}$, and therefore, $\lim _{p} \lambda\left(C_{i} V_{p}\right)=\lambda\left(C_{i}\right)$ where $\lambda$ is a right invariant Haar measure on $H_{i}$. Thus, for a large enough $p_{0}$, we have

$$
\forall h \in V_{p_{0}} \lambda\left(C_{i} h \backslash C_{i}\right)<2^{-i-k},
$$


and an easy argument using right invariance of $\lambda$ shows that we can take $V=\prod_{k<i} H_{k} \times V_{p_{0}} \times \prod_{k>i} H_{k}$.

Let $\nu_{k}$ be the normalization of $\nu_{k}^{\prime}$. Then we still have $\nu_{k} \ll \mu_{k}$ and for any $i, P_{i, C_{i}}(h, k)$ holds for all $k$ and all $h \in \pi_{i}\left(\operatorname{supp}\left(\nu_{k}\right)\right)$. Define

$$
\nu=\prod_{i} \lambda_{C_{i}} .
$$

Claim. Given $\epsilon>0$ there exists $k_{0}$ such that for all $k \geq k_{0}$ and all $n$ the following holds. If $L \subseteq \prod_{i \leq n} H_{i}$ is compact and $x \in \operatorname{supp}\left(\nu_{k}\right)$, then

$$
\left(\prod_{i \leq n} \lambda_{C_{i}}\right)\left(L \sigma_{n}(x)^{-1}\right)>\left(\prod_{i \leq n} \lambda_{C_{i}}\right)(L)-\epsilon .
$$

Proof of Claim. Take $k_{0}$ with $2^{-k_{0}+1}<\epsilon$. Let $L \subseteq \prod_{i \leq n} H_{i}$ be compact. Define $f_{j}: \prod_{i \leq j} H_{i} \rightarrow[0,1]$ for $0<j<n$ by letting

$$
f_{j}\left(\left(h_{i}\right)_{i \leq j}\right)=\left(\prod_{j<i \leq n} \lambda_{C_{i}}\right)\left(L_{\left(h_{i}\right)_{i \leq j}}\right) .
$$

Put also $f_{n}=\chi_{L}$ and $f_{0}(\emptyset)=\left(\prod_{i \leq n} \lambda_{C_{i}}\right)(L)$. With these definitions, for $0<j \leq n$ and $x \in \operatorname{supp}\left(\nu_{k}\right)$, we use the fact that $P_{j, C_{j}}\left(\pi_{j}(x), k\right)$ holds to obtain

$$
\begin{aligned}
\int_{H_{j}} & f_{j}\left(\left(h_{i}\right)_{i \leq j} \cdot \sigma_{j}(x)\right) d \lambda_{C_{j}}\left(h_{j}\right) \\
& =\int_{H_{j}} f_{j}\left(\left(h_{i}\right)_{i \leq j} \cdot\left(\sigma_{j-1}(x), 1\right) \cdot\left(1, \ldots, 1, \pi_{j}(x)\right)\right) d \lambda_{C_{j}}\left(h_{j}\right) \\
& >\int_{H_{j}} f_{j}\left(\left(h_{i}\right)_{i \leq j} \cdot\left(\sigma_{j-1}(x), 1\right)\right) d \lambda_{C_{j}}\left(h_{j}\right)-2^{-j-k} \\
& =f_{j-1}\left(\left(h_{i}\right)_{i \leq j-1} \cdot \sigma_{j-1}(x)\right)-2^{-j-k} .
\end{aligned}
$$

It follows from this calculation that

$$
\begin{aligned}
\left(\prod_{i \leq n} \lambda_{C_{i}}\right)\left(L \sigma_{n}(x)^{-1}\right) & =\int_{\prod_{i \leq n} H_{i}} f_{n}\left(\left(h_{i}\right)_{i \leq n} \cdot \sigma_{n}(x)\right) d\left(\prod_{i \leq n} \lambda_{C_{i}}\right)\left(h_{i}\right)_{i \leq n} \\
& >f_{0}(\emptyset)-2^{-k} \sum_{j \leq n} 2^{-j} \\
& >\left(\prod_{i \leq n} \lambda_{C_{i}}\right)(L)-2^{-k+1} .
\end{aligned}
$$

Thus, the conclusion of the claim holds for all $k \geq k_{0}$.

We need to show that for any compact $K \subseteq \prod_{n} H_{n}$, we have $\liminf _{k} \nu *$ $\nu_{k}(K) \geq \nu(K)$. Notice first that since $K y$ is compact for any $y \in \prod_{n} H_{n}$,

$$
\nu(K y)=\lim _{n}\left(\prod_{i \leq n} \lambda_{C_{i}}\right)\left(\sigma_{n}(K y)\right)
$$


Therefore, by the Lebesgue dominated convergence theorem, we get that for a given $\epsilon>0$ there is $n_{0}$ such that

$$
\begin{aligned}
\nu * \nu_{k}(K) & =\int_{\prod_{n} H_{n}} \nu\left(K x^{-1}\right) d \nu_{k}(x) \\
& >\int_{\prod_{n} H_{n}}\left(\prod_{i \leq n_{0}} \lambda_{C_{i}}\right)\left(\sigma_{n_{0}}(K) \sigma_{n_{0}}(x)^{-1}\right) d \nu_{k}(x)-\epsilon .
\end{aligned}
$$

Since in the above formula we can assume that $x$ ranges over $\operatorname{supp}\left(\nu_{k}\right)$, it follows from it, by Claim, that for $k$ large enough

$$
\begin{aligned}
\nu * \nu_{k}(K) & >\int_{\prod_{n} H_{n}}\left(\prod_{i \leq n_{0}} \lambda_{C_{i}}\right)\left(\sigma_{n_{0}}(K)\right) d \nu_{k}(x)-2 \epsilon \\
& =\left(\prod_{i \leq n_{0}} \lambda_{C_{i}}\right)\left(\sigma_{n_{0}}(K)\right)-2 \epsilon .
\end{aligned}
$$

Since obviously

$$
\left(\prod_{i \leq n_{0}} \lambda_{C_{i}}\right)\left(\sigma_{n_{0}}(K)\right) \geq \nu(K)
$$

(3.9) implies the conclusion of the proposition.

\section{Free subgroups AND Left HaAR Null SETS}

4.1. Left Haar null sets on groups with free subgroup at 1 . We start this subsection with some preparatory work. It will be used in the proof of Theorem 3 and in the proofs of Theorem 5 and Corollary 6 in Subsection 4.2.

Lemma 4.1. Let $G$ be a Polish group containing a non-discrete free subgroup whose all finitely generated subgroups are discrete. There exist $g_{n} \in G, n \in \mathbb{N}$, which are free generators and are such that $g_{n} \rightarrow 1$ as $n \rightarrow \infty$ and for each $n$ the subgroup generated by $\left\{g_{0}, \ldots, g_{n}\right\}$ is discrete.

Proof. Let $F<G$ be a non-discrete free subgroup of $G$ whose all finitely generated subgroups are discrete. Let $f_{m} \in G, m \in \mathbb{N}$, be free generators of $F$. We will construct the elements $g_{n}$ by induction so that $g_{n} \in F$ for each $n$. This will immediately guarantee that for each $n$ the group generated by $\left\{g_{0}, \ldots, g_{n}\right\}$ is discrete. Fix a basis of neighborhoods $1, V_{n}, n \in \mathbb{N}$, with $V_{n+1} \subseteq V_{n}$. Put $g_{0}=f_{0}$. Assume $g_{i} \in F$ for $i<n$ have been defined. Let $f_{m_{0}}$ be the first element of the sequence $\left(f_{m}\right)$ which does not appear in the reduced form of any $g_{i}, i<n$. Let $W$ be a neighborhood of 1 such that

$$
W \cup f_{m_{0}} W f_{m_{0}}^{-1} \cup f_{m_{0}}^{-1} W f_{m_{0}} \subseteq V_{n} .
$$

By non-discreteness of $F$ we can pick a nonidentity element $f$ in $F \cap W$. Let $x, y$ be the first and the last letter in the reduced form of $f$, respectively. If neither of them is in $\left\{f_{0}, \ldots, f_{m_{0}-1}\right\}$, let $g_{n}=f$. Otherwise, one of them is in 
$\left\{f_{0}, \ldots, f_{m_{0}-1}\right\}$, say it is $y$. Then, if $x \neq f_{m_{0}}$, set $g_{n}=f_{m_{0}}^{-1} f f_{m_{0}}$; if $x=f_{m_{0}}$, let $g_{n}=f_{m_{0}} f f_{m_{0}}^{-1}$. Note that this definition together with (4.1) imply that $g_{n} \in V_{n}$. Moreover, since neither the first nor the last letter in the reduced form of $g_{n}$ comes from $\left\{f_{0}, \ldots, f_{m_{0}-1}\right\}$ and each $g_{i}, i<n$, is a product of these elements, it follows that $g_{0}, \ldots, g_{n-1}, g_{n}$ are free generators.

The lemma below is, of course, a special case of the measure disintegration theorem as in [25, Theorem 4.5]. We outline its proof here for completeness and to indicate how to establish Borelness of the map $(\mu, y) \rightarrow \mu^{y}$ which may not be apparent from the argument in [25].

Lemma 4.2. Consider the space $2^{\mathbb{N}} \times \mathbb{N}$ where $\mathbb{N}$ is taken with the discrete topology. There exist Borel functions

$$
P\left(2^{\mathbb{N}} \times \mathbb{N}\right) \times 2^{\mathbb{N}} \ni(\mu, y) \rightarrow \mu^{y} \in P\left(2^{\mathbb{N}} \times \mathbb{N}\right)
$$

and

$$
P\left(2^{\mathbb{N}} \times \mathbb{N}\right) \ni \mu \rightarrow \nu_{\mu} \in P\left(2^{\mathbb{N}}\right)
$$

such that for any $y \in 2^{\mathbb{N}}$,

$$
\mu^{y}(\{(y, n): n \in \mathbb{N}\})=1
$$

and for any bounded Borel function $f: 2^{\mathbb{N}} \times \mathbb{N} \rightarrow \mathbb{R}$

$$
\int_{2^{\mathbb{N}} \times \mathbb{N}} f d \mu=\int_{2^{\mathbb{N}}}\left(\int_{2^{\mathbb{N}} \times \mathbb{N}} f d \mu^{y}\right) d \nu_{\mu}(y) .
$$

Proof. Let $\pi: 2^{\mathbb{N}} \times \mathbb{N} \rightarrow 2^{\mathbb{N}}$ be the projection. It will suffice to find a Borel function $F: P\left(2^{\mathbb{N}} \times \mathbb{N}\right) \times\left(2^{\mathbb{N}} \times \mathbb{N}\right) \rightarrow[0,1]$ such that for any bounded Borel function $f: 2^{\mathbb{N}} \times \mathbb{N} \rightarrow \mathbb{R}$, we have

$$
\int_{2^{\mathbb{N}} \times \mathbb{N}} f d \mu=\int_{2^{\mathbb{N}}}\left(\sum_{n} f(y, n) F(\mu, y, n)\right) d\left(\pi_{*} \mu\right)(y) .
$$

Assuming this has been done, we define $\mu^{y}$ by letting

$$
\mu^{y}(B)=\sum_{(y, n) \in B} F(\mu, y, n)
$$

for a Borel set $B \subseteq 2^{\mathbb{N}} \times \mathbb{N}$, and we let $\nu_{\mu}=\pi_{*} \mu$. Then we get (4.2) from (4.3), and we easily check Borelness of the maps $(\mu, y) \rightarrow \mu^{y}$ and $\mu \rightarrow \nu_{\mu}$.

To find $F$ define for $m \in \mathbb{N}$

$$
F_{m}(\mu, y, n)=\frac{\mu([y\lceil m] \times\{n\})}{\pi_{*} \mu([y\lceil m])},
$$

where $[s]$ for $s \in 2^{\{0, \ldots, m-1\}}$ stands for the set $\left\{x \in 2^{\mathbb{N}}: x \uparrow m=s\right\}$. Note that for each $m$ the function $F_{m}$ is continuous and has its values in $[0,1]$. Define

$$
F(\mu, y, n)=\limsup _{m} F_{m}(\mu, y, n) .
$$


Clearly $F$ is a Borel function. Let $\mathcal{F}_{m}$ be the (finite) $\sigma$-algebra of subsets of $2^{\mathbb{N}}$ generated by the sets $\left\{x \in 2^{\mathbb{N}}: x \uparrow(m+1)=s\right\}$ for $s \in 2^{\{0, \ldots, m\}}$. For $n \in \mathbb{N}$ let $\mu_{n}$ be the measure on $2^{\mathbb{N}}$ given by

$$
\mu_{n}(B)=\mu(B \times\{n\})
$$

for a Borel set $B \subseteq 2^{\mathbb{N}}$. Note that $\mu_{n}$ is absolutely continuous with respect to $\pi_{*} \mu$ on $\mathcal{F}_{m}$ and $2^{\mathbb{N}} \ni y \rightarrow F_{m}(\mu, y, n)$ is the corresponding Radon-Nikodym derivative. Note also that $\mathcal{F}_{m} \subseteq \mathcal{F}_{m+1}$ for each $m$ and that the $\sigma$-algebra of sets generated by $\bigcup_{m} \mathcal{F}_{m}$ is equal to the $\sigma$-algebra of all Borel subsets of $2^{\mathbb{N}}$. Thus, by [5, Theorem 35.8], given $\mu$ and $n$ the $\operatorname{limit}_{\lim _{m}} F_{m}(\mu, y, n)$ exists almost everywhere with respect to $\pi_{*} \mu$ and is equal to the Radon-Nikodym derivative of $\mu_{n}$ with respect to $\pi_{*} \mu$. Thus, $2^{\mathbb{N}} \ni y \rightarrow F(\mu, y, n)$ is equal to this Radon-Nikodym derivative. It follows now that for a bounded Borel function $f: 2^{\mathbb{N}} \times \mathbb{N} \rightarrow \mathbb{R}$, we have

$$
\begin{aligned}
\int_{2^{\mathbb{N}} \times \mathbb{N}} f d \mu & =\sum_{n} \int_{2^{\mathbb{N}}} f(y, n) d \mu_{n}(y)=\sum_{n} \int_{2^{\mathbb{N}}} f(y, n) F(\mu, y, n) d\left(\pi_{*} \mu\right)(y) \\
& =\int_{2^{\mathbb{N}}}\left(\sum_{n} f(y, n) F(\mu, y, n)\right) d\left(\pi_{*} \mu\right)(y),
\end{aligned}
$$

which is (4.3).

Lemma 4.3. Let $G$ be a Polish group. Assume that, for each $m \in \mathbb{N}, g_{1, m}, \ldots$, $g_{m, m} \in G$ are free generators and the group they generate is discrete. There exists a set $B \subseteq G \times P(G)$ such that

(i) $B$ is Borel;

(ii) $\mu\left(B^{\mu}\right)>0$ for each $\mu \in P(G)$;

(iii) for any $\mu \in P(G)$ for each $i \in \mathbb{N}$ there is an $m_{i}^{\mu} \geq i$ with $g_{i, m_{i}^{\mu}} \notin$ $\left(B^{\mu}\right)^{-1} B^{\mu}$.

Proof. Let $F_{m}$ be the subgroup of $G$ generated by $\left\{g_{n, m}: n \leq m\right\}$. Consider the left cosets of $F_{m}$ in $G$. Since $F_{m}$ is discrete, there exists a Polish space $Y_{m}$ and a Borel onto function $\phi_{m}: G \rightarrow Y_{m}$ such that $\phi_{m}\left(g_{1}\right)=\phi_{m}\left(g_{2}\right)$ precisely when $g_{1}, g_{2} \in G$ belong to the same left coset of $F_{m}$. (We can simply take $Y_{m}=G / F_{m}$ with the quotient topology.)

Let $S \subseteq G$ be a Borel set having precisely one point in common with each left coset of $F_{m}$ (see [19, Theorem 12.17]), let $f_{n}, n \in \mathbb{N}$, be a bijective enumeration of $F_{m}$, and let $\xi: 2^{\mathbb{N}} \rightarrow S$ be a Borel isomorphism (see [19, Theorem 15.6]). Then

$$
2^{\mathbb{N}} \times \mathbb{N} \ni(y, n) \rightarrow \xi(y) \cdot f_{n} \in G
$$

is a Borel isomorphism. Using it and the fact that the family of Borel subsets of $P(G)$ depends only on the family of Borel subsets of $G$ and not on the topology on $G$ (see [19, Theorem 17.24]), we can apply Lemma 4.2 to obtain, 
for each $m$, Borel functions $P(G) \times Y_{m} \ni(\mu, y) \rightarrow \mu^{y} \in P(G)$ and $P(G) \ni$ $\mu \rightarrow \nu_{\mu, m} \in P\left(Y_{m}\right)$ such that

$$
\mu^{y}\left(\phi_{m}^{-1}(y)\right)=1
$$

for $y \in Y_{m}$ and for a bounded Borel function $f: G \rightarrow \mathbb{R}$,

$$
\int_{G} f d \mu=\int_{Y_{m}}\left(\int_{\phi_{m}^{-1}(y)} f d \mu^{y}\right) d \nu_{\mu, m}(y) .
$$

We will write $G^{y}$ for $\phi_{m}^{-1}(y)$ with $y \in Y_{m}$. Since, for each $y \in Y_{m}$, $G^{y}$ is countable, $\mu^{y}$ is a nonnegative $l^{1}$ function with the support included in $G^{y}$ and the $l^{1}$ norm equal to 1 . We will, therefore, write $\mu^{y}(x)$ for $\mu^{y}(\{x\})$ for $x \in G^{y}$.

Define for $i \leq m$

$$
\begin{aligned}
A_{i, m}=\{(x, \mu) \in & G \times P(G): \\
& \left.\mu^{\phi_{m}(x)}(x)>\max \left(\mu^{\phi_{m}(x)}\left(x g_{i, m}^{-1}\right), \mu^{\phi_{m}(x)}\left(x g_{i, m}\right)\right)\right\} .
\end{aligned}
$$

Note that the set $A_{i, m}$ is Borel.

We temporarily fix $\mu \in P(G)$ and write $A_{i, m}^{\mu}$ for the set of all $x \in G$ with $(x, \mu) \in A_{i, m}$.

Note first that for all $i \leq m$,

$$
A_{i, m}^{\mu} g_{i, m}^{-1} \cap A_{i, m}^{\mu}=\emptyset \text {. }
$$

If this were not the case, there would exist $x \in A_{i, m}^{\mu}$ with $x g_{i, m} \in A_{i, m}^{\mu}$. Put $y=\phi_{m}(x)=\phi_{m}\left(x g_{i, m}\right)$. Since $x$ and $x g_{i, m}$ are elements of $A_{i, m}^{\mu}$, directly from the definition of this set, we get $\mu^{y}(x)>\mu^{y}\left(x g_{i, m}\right)$ and $\mu^{y}\left(x g_{i, m}\right)>$ $\mu^{y}\left(\left(x g_{i, m}\right) g_{i, m}^{-1}\right)=\mu^{y}(x)$, which is a contradiction.

Let us also temporarily fix $y \in Y_{m}$. Since $F_{m}$ acts freely on each $G^{y}$ by $g . x=x g^{-1}$ for $x \in G^{y}$ and $g \in F_{m}$, the Cayley graph on $F_{m}$ with respect to the generators $g_{1, m}, \ldots, g_{m, m}$ induces an isomorphic graph on each $G^{y}$ for $y \in Y_{m}$. We consider $G^{y}$ equipped with this graph. For each finite set $F \subseteq G^{y}$, we have

$$
|F| \leq \frac{1}{2 m-2}\left|\partial_{*} F\right|
$$

where $\partial_{*} F$ is the set of all edges with one vertex in $F$ and the other one outside of $F$. This inequality is well known and easy to prove by induction, see e.g. [15, Example 47 and the line preceding it]. Now Sobolev's inequality [38, Proposition 4.3 for $d=\infty]$ implies that for any $l^{1}$ function $f: G^{y} \rightarrow \mathbb{R}$, we have

$$
\|f\|_{1} \leq \frac{1}{2 m-2} \cdot \frac{1}{2} \sum_{i=1}^{m}\left(\left\|f_{g_{i, m}}-f\right\|_{1}+\left\|f_{g_{i, m}^{-1}}-f\right\|_{1}\right) .
$$

Since for all $i \leq m$

$$
\left\|f_{g_{i, m}}-f\right\|_{1}=\left\|f_{g_{i, m}^{-1}}-f\right\|_{1}
$$


we obtain that for any $l^{1}$ function $f: G^{y} \rightarrow \mathbb{R}$

$$
(2 m-2)\|f\|_{1} \leq \sum_{i=1}^{m}\left\|f_{g_{i, m}}-f\right\|_{1} .
$$

Now we let $y$ vary and, applying (4.5) to $\mu^{y}$ for all $y \in Y_{m}$, we get

$$
\begin{aligned}
2 m-2 \leq(2 m-2) \mu(G) & =\int_{Y_{m}}(2 m-2)\left\|\mu^{y}\right\|_{1} d \nu_{\mu, m}(y) \\
& \leq \sum_{i=1}^{m} \int_{Y_{m}}\left\|\mu_{g_{i, m}}^{y}-\mu^{y}\right\|_{1} d \nu_{\mu, m}(y) .
\end{aligned}
$$

This inequality can be restated to give

$$
\sum_{i=1}^{m}\left(2-\int_{Y_{m}}\left\|\mu_{g_{i, m}}^{y}-\mu^{y}\right\|_{1} d \nu_{\mu, m}(y)\right) \leq 2 .
$$

For any $y \in Y_{m}$ and any $i \leq m$ we have

$$
\begin{aligned}
& \left\|\mu_{g_{i, m}}^{y}-\mu^{y}\right\|_{1} \\
& =\sum_{\mu_{g_{i, m}}^{y}(x)>\mu^{y}(x)} \mu_{g_{i, m}}^{y}(x)-\mu^{y}(x)+\sum_{\mu^{y}(x)>\mu_{g_{i, m}}^{y}(x)} \mu^{y}(x)-\mu_{g_{i, m}}^{y}(x) \\
& \leq \mu_{g_{i, m}}^{y}\left(\left\{x \in G^{y}: \mu_{g_{i, m}}^{y}(x)>\mu^{y}(x)\right\}\right)+\mu^{y}\left(\left\{x \in G^{y}: \mu^{y}(x)>\mu_{g_{i, m}}^{y}(x)\right\}\right) \\
& =\mu^{y}\left(\left\{x \in G^{y}: \mu^{y}(x)>\mu_{g_{i, m}^{-1}}^{y}(x)\right\}\right)+\mu^{y}\left(\left\{x \in G^{y}: \mu^{y}(x)>\mu_{g_{i, m}}^{y}(x)\right\}\right) .
\end{aligned}
$$

We use the above estimate in the last inequality of the formula below to obtain

$$
\begin{aligned}
& \mu\left(G \backslash A_{i, m}^{\mu}\right) \leq\left(1-\mu\left(\left\{x \in G: \mu^{\phi_{m}(x)}(x)>\mu_{g_{i, m}^{-1}}^{\phi_{m}(x)}(x)\right\}\right)\right) \\
&+\left(1-\mu\left(\left\{x \in G: \mu^{\phi_{m}(x)}(x)>\mu_{g_{i, m}}^{\phi_{m}(x)}(x)\right\}\right)\right) \\
&=2-\left(\int_{Y_{m}} \mu^{y}\left(\left\{x \in G^{y}: \mu^{y}(x)>\mu_{g_{i, m}^{-1}}^{y}(x)\right\}\right)\right. \\
&\left.\quad+\mu^{y}\left(\left\{x \in G^{y}: \mu^{y}(x)>\mu_{g_{i, m}}^{y}(x)\right\}\right) d \nu_{m}(y)\right) \\
& \leq 2-\int_{Y_{m}}\left\|\mu_{g_{i, m}}^{y}-\mu^{y}\right\|_{1} d \nu_{m}(y) .
\end{aligned}
$$

It follows from (4.7) and (4.6) that for each $m$

$$
\sum_{i=1}^{m} \mu\left(G \backslash A_{i, m}^{\mu}\right) \leq 2 .
$$

Now we let $\mu$ vary. Since $A_{i, m}$ is Borel, the function

$$
P(G) \ni \mu \rightarrow \mu\left(G \backslash A_{i, m}^{\mu}\right) \in \mathbb{R}
$$


is Borel, see [19, Theorem 17.25]. It follows, therefore, that the assignment $P(G) \ni \mu \rightarrow m_{i}^{\mu} \in \mathbb{N}$ given by

$$
m_{i}^{\mu}=\min \left\{n: \mu\left(G \backslash A_{i, n}^{\mu}\right) \leq \inf _{m \geq i} \mu\left(G \backslash A_{i, m}^{\mu}\right)+2^{-i-1}\right\}
$$

is Borel. We claim that for each $\mu \in P(G)$

$$
\sum_{i=1}^{\infty} \mu\left(G \backslash A_{i, m_{i}^{\mu}}^{\mu}\right) \leq 3
$$

Indeed, given $k$ let $M_{k}=\max \left(k, \max _{i \leq k} m_{i}^{\mu}\right)$. Then by (4.8)

$$
\sum_{i=1}^{k} \mu\left(G \backslash A_{i, m_{i}^{\mu}}^{\mu}\right) \leq \sum_{i=1}^{M_{k}}\left(\mu\left(G \backslash A_{i, M_{k}}^{\mu}\right)+2^{-i-1}\right) \leq 2+\sum_{i=1}^{M_{k}} 2^{-i-1}<3
$$

and (4.10) follows.

Define now for $j \geq 2$

$$
\begin{aligned}
& E_{1}=P(G) \backslash\left\{\mu \in P(G): \sum_{i=1}^{\infty} \mu\left(G \backslash A_{i, m_{i}^{\mu}}^{\mu}\right)<1\right\} \\
& E_{j}=\left\{\mu \in P(G): \sum_{i=j-1}^{\infty} \mu\left(G \backslash A_{i, m_{i}^{\mu}}^{\mu}\right) \geq 1\right. \text { and } \\
& \left.\qquad \sum_{i=j}^{\infty} \mu\left(G \backslash A_{i, m_{i}^{\mu}}^{\mu}\right)<1\right\} .
\end{aligned}
$$

By Borelness of the function (4.9) and of the assignment $\mu \rightarrow m_{i}^{\mu}$, the sets $E_{j}$ are Borel. By (4.10) we have $\bigcup_{j} E_{j}=P(G)$.

Fix $j$. Define

$$
A_{j}=\left\{(x, \mu) \in G \times E_{j}: x \in \bigcap_{i \geq j} A_{i, m_{i}^{\mu}}^{\mu}\right\} .
$$

Note that $A_{j}$ is Borel. Equation (4.4) implies that for any $\mu \in E_{j}$

$$
\left(A_{j}^{\mu}\right)^{-1} A_{j}^{\mu} \cap\left\{g_{i, m_{i}^{\mu}}: i \geq j\right\}=\emptyset .
$$

From the definition (4.11) of the sets $E_{j}$ it follows that for any $\mu \in E_{j}$, $\sum_{i=j}^{\infty} \mu\left(G \backslash A_{i, m_{i}^{\mu}}^{\mu}\right)<1$ and, therefore, $\mu\left(A_{j}^{\mu}\right)>0$. Let $V$ be an open neighborhood of $1 \in G$ such that $g_{i, i} \notin V^{-1} V$ for all $i<j$. Cover $G$ by countably many left translates $V_{n}, n \in \mathbb{N}$, of $V$. Now the function

$$
E_{j} \ni \mu \rightarrow n_{\mu}=\min \left\{n: \mu\left(A_{j}^{\mu} \cap V_{n}\right)>0\right\} \in \mathbb{N}
$$

is well defined since $\mu\left(A_{j}^{\mu}\right)>0$ and is Borel since the set $A_{j} \cap\left(V_{n} \times E_{j}\right)$ is Borel for each $n$, see [19, Theorem 17.25]. Thus,

$$
B_{j}=A_{j} \cap\left\{(x, \mu) \in G \times E_{j}: x \in V_{n_{\mu}}\right\}
$$

is Borel and such that 
(a) $B_{j} \subseteq A_{j}$;

(b) $\mu\left(B_{j}^{\mu}\right)>0$ for $\mu \in E_{j}$;

(c) $\left(B_{j}^{\mu}\right)^{-1} B_{j}^{\mu} \cap\left\{g_{i, i}: i<j\right\}=\emptyset$.

Note further that (a) and (4.12) imply that $\left(B_{j}^{\mu}\right)^{-1} B_{j}^{\mu} \cap\left\{g_{i, m_{i}^{\mu}}: i \geq j\right\}=\emptyset$. This together with (c) gives that for each $i$ there exists $m_{i} \geq i$ with $g_{i, m_{i}} \notin$ $\left(B_{j}^{\mu}\right)^{-1} B_{j}^{\mu}$. This, combined with (b), implies that $B_{j}$ is a Borel set fulfilling requirements (ii) and (iii) from the statement of the lemma for $\mu \in E_{j}$.

Define

$$
B=\bigcup_{j} B_{j}
$$

Clearly, $B$ is as required: it fulfills (ii) and (iii) by what was said above and is Borel since each $B_{j}$ is.

The assumption of strong non-local compactness in Theorem 3(ii) will be used in the proof of this theorem through the following lemma.

Lemma 4.4. Let $G$ be a Polish group which is strongly non-locally compact. Let $X$ be a Borel subset of a Polish space and let $F: X \rightarrow \mathcal{K}(G)$ be Borel. Then there exists a Borel function $X \ni x \rightarrow g_{x} \in G$ such that for any distinct $x_{1}, x_{2} \in X, g_{x_{1}} F\left(x_{1}\right) \cap g_{x_{2}} F\left(x_{2}\right)=\emptyset$.

Proof. Let $\mathbb{N}<\mathbb{N}$ stand for the set of all finite sequences of natural numbers. We think of $s \in \mathbb{N}<\mathbb{N}$ as a function $s:\{0, \ldots, n-1\} \rightarrow \mathbb{N}$ for some $n \in \mathbb{N}$ and write $|s|$ for $n$. If $m \leq|s|$, we let $s \uparrow m$ be the restriction of $s$ to $\{0, \ldots, m-1\}$. For $s$ as above and for $k \in \mathbb{N}$, let $s^{\wedge} k$ stand for an element of $\mathbb{N}<\mathbb{N}$ such that $\left(s^{\wedge} k\right) \uparrow|s|=s$ and $\left(s^{\wedge} k\right)(|s|)=k$.

Let us fix $F$ and $X$ as in the statement. By making the topology on $X$ stronger while keeping the Borel $\sigma$-algebra unchanged, we can assume that $F$ is continuous and that $X$ is Polish and zero dimensional [19, 13.1, 13.11, 13.5]. We fix a complete metric $d$ on $X$ bounded by 1 . We also fix a complete metric $\rho_{1}$ is bounded by 1 and a left invariant metric $\rho_{2}$ on $G$ bounded by $1 / 2$. Note that in general $\rho_{1} \neq \rho_{2}$.

We will construct $V_{s} \subseteq X$ closed and open, $W_{s}, U_{s} \subseteq G$ open neighborhoods of $1, g_{s} \in G$, and $r_{s}$ positive real numbers for $s \in \mathbb{N}<\mathbb{N}$. Moreover, for $s \in \mathbb{N}<\mathbb{N}$ with $|s|>0$, we define $h_{i, s} \in G$, for $i \leq m_{s}$ for some $m_{s} \in \mathbb{N}$. They will have the following properties for all $s \in \mathbb{N}<\mathbb{N}$ in points (i), (vi), (viii)-(x) and and for all $s \in \mathbb{N}<\mathbb{N}$ with $|s|>0$ in points (ii)-(v), (vii)

(i) $V_{\emptyset}=X$ and $V_{s}$ is partitioned by $\left\{V_{s^{\wedge} n}: n \in \mathbb{N}\right\}$;

(ii) for all $x \in V_{s}, F(x) \subseteq C_{s}$ where $C_{s}=\bigcup_{i \leq m_{s}} h_{i, s} U_{s \||s|-1}$;

(iii) $g_{\emptyset}=1$ and $g_{s} \in W_{s \uparrow|s|-1}$;

(iv) $g_{s} B_{s} \cap g_{t} B_{t}=\emptyset$ if $|s|=|t|$ and $s$ and $t$ differ only on the last digit where $B_{s}=\bigcup_{i \leq m_{s}} h_{i, s} U_{s \uparrow|s|-1}^{2}$; 
(v) $h_{i, s}^{-1} W_{s} h_{i, s} \subseteq U_{s \||s|-1}$ for each $i \leq m_{s}$;

(vi) $U_{s} \subseteq W_{s}$ and $W_{s}$ cannot be covered by finitely many two-sided translates of $U_{s}^{2} U_{s}^{-2}$;

(vii) $\left\{g \in G: \rho_{2}\left(g, g_{s}\right)<r_{s}\right\} \subseteq W_{s \||s|-1}$;

(viii) $\rho_{1}-\operatorname{diam}\left(g_{s}\left\lceil 0, g_{s} W_{s}\right)<2^{-|s|}\right.$;

(ix) $\rho_{2}$-diam $\left(W_{s}\right)<\frac{r_{s \mid k}}{2^{|s|-k+1}}$ for all $k \leq|s|$;

(x) $d$-diam $\left(V_{s}\right) \leq \frac{1}{|s|+1}$.

A construction of the objects with the properties listed above goes as follows. Let $V_{\emptyset}=X, g_{\emptyset}=1, W_{\emptyset}=G$, and $r_{\emptyset}=1$. Strong non-local compactness of $G$ allows us to pick $U_{\emptyset}$ so that (vi) holds for it. Thus, conditions (i), (vi), (viii)(x) and $g_{\emptyset}=1$ in (iii) are fulfilled for $s=\emptyset$. The other conditions involve $s \in \mathbb{N}<\mathbb{N}$ with $|s| \geq 1$.

Now assume $V_{s}$ is given. We find $V_{s^{\wedge} n}, m_{s^{\wedge} n}$, and $h_{i, s^{\wedge} n}$ for $i \leq m_{s^{\wedge} n}$ with (i), (ii), (x). In order to do this, cover $F\left(V_{s}\right)$ with sets of the form

$$
\left\{K \in \mathcal{K}(G): K \subseteq h_{0} U_{s} \cup \cdots \cup h_{m} U_{s}\right\}
$$

for some $h_{0}, \ldots, h_{m} \in G$. Now find $V_{s^{\wedge} n}$ so that (i) nd (x) are fulfilled and for each $n, F\left(V_{s^{\wedge} n}\right)$ is included in a set of the form (4.13). This uses continuity of $F$ and zero dimensionality of $X$. Now for each $n$ pick $m_{s^{\wedge} n} \in \mathbb{N}$ and $h_{i, s^{\wedge} n}$ for $i \leq m_{s^{\wedge} n}$ so that

$$
F\left(V_{s^{\wedge} n}\right) \subseteq\left\{K \in \mathcal{K}(G): K \subseteq h_{0, s^{\wedge} n} U_{s} \cup \cdots \cup h_{m_{s^{\wedge} n}, s^{\wedge} n} U_{s}\right\} .
$$

Note that we have at this point the sets $C_{s^{\wedge} n}$ and $B_{s^{\wedge} n}$ as in points (ii) and (iv). Now we find elements $g_{s^{\wedge} n}$ with (iii) and (iv) by recursion on $n$. Pick $g_{s^{\wedge} 0} \in W_{s}$. Assume $g_{s^{\wedge} k}$ have been defined for $k \leq n$. Consider the set

$$
g_{s^{\wedge} 0} B_{s^{\wedge} 0} B_{s^{\wedge}(n+1)}^{-1} \cup \cdots \cup g_{s^{\wedge} n} B_{s^{\wedge} n} B_{s^{\wedge}(n+1)}^{-1} .
$$

Note that this set is a finite union of two sided translations of $U_{s}^{2} U_{s}^{-2}$. Thus, it does not contain $W_{s}$ by (vi) for $s$, so we can pick $g_{s^{\wedge}(n+1)} \in W_{s}$ not in the above set. Then, obviously, for any $k \leq n$

$$
g_{s^{\wedge}(n+1)} B_{s^{\wedge}(n+1)} \cap g_{s^{\wedge} k} B_{s^{\wedge} k}=\emptyset .
$$

Let now $r_{s^{\wedge} n}>0$ be such that (vii) holds. Pick $W_{s^{\wedge} n} \ni 1$ small enough to fulfill (v), (viii), (ix). Then, using the fact that $G$ is strongly non-locally compact, find $U_{s^{\wedge} n}$ satisfying (vi). Thus, conditions (i)-(x) hold for the newly constructed objects.

For each $x \in X$ let $\eta_{x}$ be the unique element of $\mathbb{N}^{\mathbb{N}}$ such that $x \in V_{\eta_{x} \mid n}$ for each $n$. Define

$$
g_{x}=g_{\eta_{x}\lceil 0} g_{\eta_{x} \uparrow 1} \cdots
$$


The product converges by (iii) and (viii) since $\rho_{1}$ is complete. In fact, since $X \ni x \rightarrow \eta_{x} \in \mathbb{N}^{\mathbb{N}}$ is clearly continuous, (iii) and (vii) give that the function $X \ni x \rightarrow g_{x} \in G$ is continuous.

Now, for any $s \in \mathbb{N}<\mathbb{N}$ we have

(a) $g_{s\lceil 0} \cdots g_{s} g_{s^{\wedge} k_{1}} B_{s^{\wedge} k_{1}} \cap g_{s\lceil 0} \cdots g_{s} g_{s^{\wedge} k_{2}} B_{s^{\wedge} k_{2}}=\emptyset$ if $k_{1} \neq k_{2}$;

(b) $g_{x} C_{s} \subseteq g_{s\lceil 0} \cdots g_{s} B_{s}$ if $s \subseteq \eta_{x}$ with $|s|>0$.

Point (a) follows from (iv). To see (b), put $s^{\wedge} n=\eta_{x} \uparrow|s|+1$. Note that by left invariance of $\rho_{2}$ we have

$$
\rho_{2}\left(g_{\eta_{x}|| s \mid+1} g_{\eta_{x}|| s \mid+2} \cdots, g_{\eta_{x}|| s \mid+1}\right) \leq \sum_{i=|s|+2}^{\infty} \rho_{2}\left(g_{\eta_{x} \backslash i}, 1\right) .
$$

Point (iii) gives for all $i \geq|s|+2, g_{\eta_{x} \uparrow i} \in W_{\eta_{x} \uparrow i-1}$ which, together with (ix), implies that

$$
\rho_{2}\left(g_{\eta_{x} \uparrow i}, 1\right)<\frac{r_{s^{\wedge} n}}{2^{(i-1)-(|s|+1)+1}}=\frac{r_{s^{\wedge} n}}{2^{i-|s|-1}}
$$

whereby we obtain

$$
\rho_{2}\left(g_{\eta_{x} \||s|+1} g_{\eta_{x} \||s|+2} \cdots, g_{\eta_{x} \||s|+1}\right)<\sum_{i=|s|+2}^{\infty} \frac{r_{s^{\wedge} n}}{2^{i-|s|-1}}=r_{s^{\wedge} n} .
$$

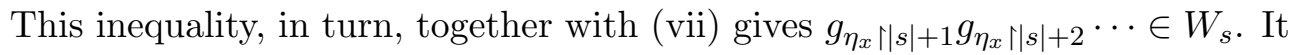
follows from this, (v) and the definitions

$$
C_{s}=\bigcup_{i \leq m_{s}} h_{i, s} U_{s \||s|-1} \text { and } B_{s}=\bigcup_{i \leq m_{s}} h_{i, s} U_{s \||s|-1}^{2}
$$

that

$$
g_{\eta_{x} \||s|+1} g_{\eta_{x} \| s \mid+2} \cdots C_{s} \subseteq B_{s}
$$

Thus, we obtain

$$
g_{x} C_{s}=g_{s \uparrow 0} \cdots g_{s}\left(g_{\eta_{x} \uparrow|s|+1} g_{\eta_{x} \uparrow|s|+2} \cdots C_{s}\right) \subseteq g_{s\lceil 0} \cdots g_{s} B_{s}
$$

which is (b).

Since $F(x) \subseteq C_{\eta_{x} \mid n}$ for each $n$, it follows from (b) that if $x \neq y$ and $i$ is the first natural number with $\eta_{x}(i) \neq \eta_{y}(i)$, then, if we let $s=\eta_{x}\left\lceil i=\eta_{y} \uparrow i\right.$, $g_{x} F(x) \subseteq g_{s\lceil 0} \cdots g_{s} g_{s^{\wedge} \eta_{x}(i)} B_{s^{\wedge} \eta_{x}(i)}$ and $g_{y} F(y) \subseteq g_{s \mid 0} \cdots g_{s} g_{s^{\wedge} \eta_{y}(i)} B_{s^{\wedge} \eta_{y}(i)}$. In light of (a) this immediately gives, $g_{x} F(x) \cap g_{y} F(y)=\emptyset$.

Proof of Theorem 3. (i) The proof of this point uses only Lemma 4.1. This lemma guarantees the existence of a sequence $\left(g_{n}\right)$ converging to 1 whose elements are free generators of the subgroup they generate. By going to a further subsequence we can find $a_{n}^{0}, a_{n}^{1} \in G$ such that

(a) for each $n, a_{i}^{0}, a_{i}^{1}$ for $i \leq n$ are free generators and the subgroup generated by them is discrete; 
(b) for any $x \in 2^{\mathbb{N}}$, the infinite product $\cdots a_{2}^{x(2)} a_{1}^{x(1)} a_{0}^{x(0)}$ converges and for any neighborhood $U$ of 1 there exists $n \in \mathbb{N}$ such that for any $x \in 2^{\mathbb{N}}$

$$
\cdots a_{n+2}^{x(n+2)} a_{n+1}^{x(n+1)} a_{n}^{x(n)} \in U .
$$

Point (a) is fulfilled if only $\left(a_{n}^{0}\right)$ and $\left(a_{n}^{1}\right)$ are subsequences of $\left(g_{n}\right)$ and $a_{n_{1}}^{0} \neq a_{n_{2}}^{1}$ for all $n_{1}, n_{2} \in \mathbb{N}$. Point (b) can be fulfilled by making $\left(a_{n}^{0}\right)$ and $\left(a_{n}^{1}\right)$ converge to 1 fast enough.

Let $H_{n}$ be the subgroup of $G$ generated by $\left\{a_{i}^{0}, a_{i}^{1}: i \leq n\right\}$. Since by (a) $H_{n}$ is discrete, we can find $S_{n} \subseteq G$ a Borel set which is a selector for the family of all left cosets of $H_{n}$, see [19, Theorem 12.17]. Thus, for any $g \in G$ there exist unique $s_{n}^{g} \in S_{n}$ and $f_{n}^{g} \in H_{n}$ such that $g=s_{n}^{g} f_{n}^{g}$. Since for $f \in H_{n}$

$$
\begin{aligned}
f_{n}^{g}=f & \Leftrightarrow \forall s \in S_{n}\left(s^{-1} g \in H_{n} \Rightarrow s^{-1} g=f\right) \\
& \Leftrightarrow \exists s \in S_{n} s^{-1} g=f,
\end{aligned}
$$

it follows that $\left\{g \in G: f_{n}^{g}=f\right\}$ is both co-analytic and analytic; thus, from the Suslin separation theorem [19, Theorem 14.11], we get that the function $G \ni g \rightarrow f_{n}^{g} \in H_{n}$ is Borel, where $H_{n}$ is taken with the discrete topology. By (a), we can think of elements of $H_{n}$ as irreducible words in the alphabet $\left\{a_{i}^{0}, a_{i}^{1},\left(a_{i}^{0}\right)^{-1},\left(a_{i}^{1}\right)^{-1}: i \leq n\right\}$. This allows us to make the following definition. If $f_{n}^{g} \neq 1$, let

$$
t_{n}(g)=\text { the last letter in } f_{n}^{g},
$$

if $f_{n}^{g}=1$, let $t_{n}(g)=1$. Note that the function $G \ni g \rightarrow t_{n}(g)$ is Borel since $g \rightarrow f_{n}^{g}$ is Borel. Define now

$$
\begin{aligned}
& B=\left\{g \in G: t_{n}(g) \neq a_{0}^{0} \text { for infinitely many } n\right\} \\
& C=\left\{g \in G: t_{n}(g) \neq a_{0}^{1} \text { for infinitely many } n\right\} .
\end{aligned}
$$

Note that $C \subseteq B a_{0}^{1}$. Indeed, if $g \in C$, then for infinitely many $n$ we have $t_{n}(g) \neq a_{0}^{1}$, so $t_{n}\left(g\left(a_{0}^{1}\right)^{-1}\right)=\left(a_{0}^{1}\right)^{-1}$ for infinitely many $n$, whence $g\left(a_{0}^{1}\right)^{-1} \in B$. Thus, it will suffice to see that $B$ is Borel, that $B \cup C=G$, and that $B$ is left Haar null. Borelness of $t_{n}$ implies that $B$ is Borel. If $g \in G \backslash(B \cup C)$, then $t_{n}(g)=a_{0}^{0}$ for all $n$ large enough and $t_{n}(g)=a_{0}^{1}$ for all $n$ large enough which leads to a contradiction as $a_{0}^{0} \neq a_{0}^{1}$. Thus, $B \cup C=G$. Therefore, it remains to show that $B$ is left Haar null.

Consider the function $\phi: 2^{\mathbb{N}} \rightarrow G$ given by

$$
\phi(x)=\cdots a_{2}^{x(1)} a_{1}^{x(0)} a_{0}^{0} .
$$

By (b), $\phi$ is well defined and continuous. Therefore, we can define a Borel probability measure $\mu$ on $G$ by letting for any Borel set $A \subseteq G$

$$
\mu(A)=\lambda\left(\phi^{-1}(A)\right)
$$


where $\lambda$ is the product measure on $2^{\mathbb{N}}=\{0,1\}^{\mathbb{N}}$ arising from the measures assigning weight $1 / 2$ to both 0 and 1 on each coordinate. We claim that for any $h \in G, \mu\left(h^{-1} B\right)=0$ which makes $B$ left Haar null.

Fix $h \in G$. We need to show that

$$
\lambda\left(\left\{x \in 2^{\mathbb{N}}: h\left(\cdots a_{2}^{x(1)} a_{1}^{x(0)} a_{0}^{0}\right) \in B\right\}\right)=0 .
$$

Note that, by the definition of $B$, it will suffice to show that for each $n \in \mathbb{N}$

$$
\lambda\left(\left\{x \in 2^{\mathbb{N}}: t_{n}\left(h\left(\cdots a_{2}^{x(1)} a_{1}^{x(0)} a_{0}^{0}\right)\right) \neq a_{0}^{0}\right\}\right) \leq 2^{-n} .
$$

Fix $n \in \mathbb{N}$. Note that $\lambda$ is the product of a Borel probability measure on $2^{\mathbb{N} \backslash\{0, \ldots, n-1\}}$ and the normalization of the counting measure on $2^{\{0, \ldots, n-1\}}$. Therefore, by Fubini's theorem, to see (4.14), it suffices to check that for any $z \in 2^{\mathbb{N} \backslash\{0, \ldots, n-1\}}$

$$
\begin{aligned}
& \mid\left\{\sigma \in 2^{\{0, \ldots, n-1\}}:\right. \\
& \left.\quad t_{n}\left(h\left(\cdots a_{n+2}^{z(n+1)} a_{n+1}^{z(n)}\right)\left(a_{n}^{\sigma(n-1)} \cdots a_{1}^{\sigma(0)} a_{0}^{0}\right)\right) \neq a_{0}^{0}\right\} \mid \leq 1 .
\end{aligned}
$$

Fix a $z \in 2^{\mathbb{N} \backslash\{0, \ldots, n-1\}}$. Find the unique $s_{n}^{z} \in S_{n}$ and $f_{n}^{z} \in H_{n}$ with

$$
s_{n}^{z} f_{n}^{z}=h\left(\cdots a_{n+2}^{z(n+1)} a_{n+1}^{z(n)}\right) .
$$

Note that for $\sigma \in 2^{\{0, \ldots, n-1\}}$ the element $a_{n}^{\sigma(n-1)} \cdots a_{1}^{\sigma(0)} a_{0}^{0}$ is in $H_{n}$ and, therefore, by (4.16), $t_{n}\left(h\left(\cdots a_{n+2}^{z(n+1)} a_{n+1}^{z(n)}\right)\left(a_{n}^{\sigma(n-1)} \cdots a_{1}^{\sigma(0)} a_{0}^{0}\right)\right)$ is simply the last letter in the reduced form of $f_{n}^{z} a_{n}^{\sigma(n-1)} \cdots a_{1}^{\sigma(0)} a_{0}^{0}$. This last letter is not equal to $a_{0}^{0}$ only if $f_{n}^{z}$ ends in $\left(a_{0}^{0}\right)^{-1}\left(a_{1}^{\sigma(0)}\right)^{-1} \cdots\left(a_{n}^{\sigma(n-1)}\right)^{-1}$. Since $f_{n}^{z}$ does not depend on $\sigma$, this can happen for at most one $\sigma$ confirming (4.15).

(ii) Let us fix a sequence $\left(g_{n}\right)$ as given by Lemma 4.1. We will produce a Borel non-left Haar null set $A \subseteq G$ such that $g_{n} \notin A^{-1} A$ for all $n \in \mathbb{N}$. Since $g_{n} \rightarrow 1$, this will give that 1 is not in the interior of $A^{-1} A$ proving (ii) of Theorem 3.

Let

$$
X=\{(\mu, K) \in P(G) \times \mathcal{K}(G): \mu(K)>0\} .
$$

Then $X$ is a Borel subset of the Polish space $P(G) \times \mathcal{K}(G)$, see $[19,17.29]$. For $x \in X$, we will write $x=\left(\mu_{x}, K_{x}\right)$. Let $L_{0}=\{1\} \cup\left\{g_{n}: n \in \mathbb{N}\right\}$. Then $L_{0}$ is compact and the map $\mathcal{K}(G) \ni K \rightarrow K L_{0} \in \mathcal{K}(G)$ is easily seen to be Borel. Note that if $K_{1} L_{0} \cap K_{2} L_{0}=\emptyset$, then $K_{2}^{-1} K_{1} \cap L_{0}=\emptyset$. Therefore, by Lemma 4.4 applied to the Borel map $F: X \rightarrow \mathcal{K}(G)$ given by

$$
F(x)=K_{x} L_{0},
$$

we obtain a Borel function $X \ni x \rightarrow g_{x} \in G$ such that

$$
\forall x_{1}, x_{2} \in X \text { if } x_{1} \neq x_{2} \text {, then }\left(\left(g_{x_{1}} K_{x_{1}}\right)^{-1} g_{x_{2}} K_{x_{2}}\right) \cap L_{0}=\emptyset .
$$


Let $H: X \rightarrow P(G)$ be given by

$$
H(x)=\frac{1}{\mu\left(K_{x}\right)}\left(\mu_{x}\lceil K) .\right.
$$

The function $H$ is easily seen to be Borel. Let $B \subseteq G \times P(G)$ be a Borel set as in Lemma 4.3 produced for the sequences given by $g_{i, m}=g_{i}$ for $i \leq m \in \mathbb{N}$. Define now

$$
B_{1}=\{(g, x) \in G \times X:(g, H(x)) \in B\}
$$

which is Borel by Borelness of $B$ and $H$. The set

$$
C=\left\{(g, x) \in G \times X: g \in K_{x}\right\}
$$

is closed and, therefore,

$$
B_{2}=B_{1} \cap C
$$

is Borel. Note that $B_{2}$ has the following properties. For each $x \in X$,

(1) $B_{2}^{x} \subseteq K_{x}$,

(2) $\mu_{x}\left(B_{2}^{x}\right)>0$,

(3) for each $i$ there exists $m_{i}^{x} \geq i$ such that $g_{i, m_{i}^{x}} \notin\left(B_{2}^{x}\right)^{-1}\left(B_{2}\right)_{x}$.

Note, however, that for each $i$ and $m \geq i, g_{i, m}=g_{i}$. Thus, condition (3) implies that for any $x \in X$

$$
\left(B_{2}^{x}\right)^{-1} B_{2}^{x} \cap\left\{g_{n}: n \in \mathbb{N}\right\}=\emptyset
$$

and hence

$$
\left(g_{x} B_{2}^{x}\right)^{-1} g_{x} B_{2}^{x} \cap\left\{g_{n}: n \in \mathbb{N}\right\}=\emptyset
$$

Let now $A$ be the image of $B_{2}$ under the Borel map $X \times G \rightarrow G$ given by $(x, g) \rightarrow g_{x} g$. This map is injective on $B_{2}$. Indeed, since $L_{0}$ contains 1 , (4.17) gives $g_{x_{1}} K_{x_{1}} \cap g_{x_{2}} K_{x_{2}}=\emptyset$ for $x_{1} \neq x_{2}$, which together with (1) imply injectivity of $(x, g) \rightarrow g_{x} g$ on $B_{2}$. Thus, $A$ is Borel by [19, Theorem 15.1]. Clearly, $A=\bigcup_{x \in X} g_{x}\left(B_{2}^{x}\right)$. Therefore, it is immediate from (2) that $A$ is not left Haar null. Furthermore,

$$
A^{-1} A=\bigcup_{x \in X}\left(g_{x} B_{2}^{x}\right)^{-1} g_{x} B_{2}^{x} \cup \bigcup_{x_{1} \neq x_{2} \in X}\left(g_{x_{1}} B_{2}^{x_{1}}\right)^{-1} g_{x_{2}} B_{2}^{x_{2}}
$$

hence by (4.18) and (4.17) and (1) it follows that

$$
A^{-1} A \cap\left\{g_{n}: n \in \mathbb{N}\right\}=\emptyset .
$$


4.2. Consequences for locally compact groups and Haar null sets. We deduce now Theorem 5 from Lemma 4.3.

Proof of Theorem 5. Assume towards contradiction that each neighborhood $V$ of 1 has two elements $x, y$ generating a discrete, non-Abelian, free subgroup. Note that the two elements are then free generators, see [13, Theorem 7.3.3]. Since as is well known for each $n \in \mathbb{N}$ the free group on two generators contains a subgroup which is a free group on $n$ generators, there exist $n$ words $w_{1}, \ldots, w_{n}$ in two letters which when evaluated on $x$ and $y$ give $n$ free generators. Thus, $\bigcup_{i \leq n} w_{i}(V \times V)$ contains $n$ free generators generating a discrete subgroup. Since $V$ is an arbitrary neighborhood of 1 , it follows that each neighborhood of 1 contains $n$ free generators generating a discrete subgroup.

Fix an open basis $V_{n}, n \in \mathbb{N}$, at 1 with $V_{n+1} \subseteq V_{n}$. For each $n$, let $g_{1, n}, \ldots g_{n, n} \in V_{n}$ be free generators of a discrete subgroup of $G$. Let $B \subseteq$ $G \times P(G)$ be a Borel set constructed in Lemma 4.3 for these elements $g_{i, n}$. Since the left Haar measure on $G$ is $\sigma$-finite, there exists a Borel probability measure $\mu$ on $G$ whose measure zero sets are precisely Haar measure zero sets. By Lemma 4.3(ii), $\mu\left(B^{\mu}\right)>0$, whence $B^{\mu}$ has a positive Haar measure. Also by (iii) of the same lemma, for each $i$ there exists an $m_{i} \geq i$ such that $g_{i, m_{i}} \notin\left(B^{\mu}\right)^{-1} B^{\mu}$ for each $i$. Since $g_{i, m_{i}} \rightarrow 1$ as $i \rightarrow \infty$, it follows that 1 is not in the interior of $\left(B^{\mu}\right)^{-1} B^{\mu}$ contradicting [16, 20.17].

We present now the proof of Corollary 6 from Theorem 3 .

Proof of Corollary 6. (i) The set $B$ in Theorem 3(i) is left Haar null but not Haar null. In fact, it is not even right Haar null. Otherwise, the Borel probability measure witnessing that it is right Haar null would assign measure 0 to $B \cup B g$ for any $g \in G$ and, therefore, it would assign measure 0 to $G$, contradiction.

(ii) This is immediate from Theorem 3(ii) and the fact that Haar null sets are left Haar null.

\subsection{Strongly non-locally compact groups with a free subgroup at 1 .} In this section, we will present examples of Polish groups to which Theorem 3 and Corollaries 4 and 6 apply. We will start with developing criteria for having a free subgroup at 1 and for strong non-local compactness.

\subsubsection{A criterium for having a free subgroup at 1 .}

Proposition 4.1. Let $H_{n}, n \in \mathbb{N}$, be Polish groups infinitely many of which contain a discrete non-Abelian free group. Assume $G$ is a Polish group containing a closed subgroup isomorphic to $\prod_{n} H_{n}$. Then $G$ has a free subgroup at 1 . 
Proof. It suffices to prove it for $G=\prod_{n} H_{n}$ assuming that all $H_{n}$ contain discrete non-Abelian subgroups. We can also assume that each $H_{n}$ contains a discrete non-Abelian free subgroup. Since a non-Abelian free subgroup contains a free subgroup $F_{n}$ on $n$ generators for $n \in \mathbb{N}$, we can assume that $F_{n}<H_{n}$. Let $f_{i, n}, i<n$, be generators of $F_{n}$. Define $h_{m} \in \prod_{n} H_{n}$ by letting

$$
\begin{aligned}
& h_{m}(n)=1 \text { if } n \leq m \\
& h_{m}(n)=f_{m, n} \text { if } n>m
\end{aligned}
$$

Since the group generated by $\left\{h_{m}: m \leq n\right\}$ projects injectively onto $F_{n}$, we see that it is free and discrete, and the proposition follows.

4.3.2. Criteria for strong non-local compactness. The obvious proposition below justifies the name of strong non-local compactness. We leave its proof to the reader.

Proposition 4.2. If a Polish group is strongly non-locally compact, then it is not locally compact.

The next two propositions give criteria for strong non-local compactness.

Proposition 4.3. $\quad$ (i) If $G$ is a Polish group which has a two-sided invariant metric and is not locally compact, then it is strongly non-locally compact.

(ii) If $G$ is a Polish group which is the product of a sequence of Polish non-compact groups, then $G$ is strongly non-locally compact.

Proof. To see (i) fix a two-sided invariant metric $d$ on $G$. Since $G$ is Polish, any two-sided invariant metric, in particular $d$, is complete, see [17, Lemma 7.4]. Now if $G$ is not strongly non-locally compact, then there is a neighborhood $U$ of 1 which can be covered by $F_{1} V F_{2}$ for any neighborhood $V$ of 1 and for some finite $F_{1}, F_{2} \subseteq G$. Since $d$ is two-sided invariant, it follows that $U$ is totally bounded with respect to $d$. Since $d$ is complete, $\bar{U}$ is compact.

To see (ii), let $G=\prod_{n} H_{n}$ with $H_{n}$ Polish for $n \in \mathbb{N}$. If $G$ is not strongly non-locally compact, fix a neighborhood $U$ of 1 which can be covered by $F_{1} V F_{2}$ for any neighborhood $V$ of 1 and for some finite sets $F_{1}, F_{2} \subseteq G$. We can assume that $U=\prod_{n<N} U_{n} \times \prod_{n \geq N} H_{n}$ for some open $U_{n} \subseteq H_{n}$. Since $V$ can be taken to be $\prod_{n<N} H_{n} \times V_{N} \times \prod_{n>N} H_{n}$ with $V_{N}$ an arbitrary open neighborhood of 1 in $H_{N}$, it follows that $H_{N}$ can be covered by finitely many two-sided translates of each neighborhood of 1 . This implies, by [31, Lemma $1.2]$, that $H_{N}$ is compact.

Let a Polish group $G$ act continuously on a metric space $(X, d)$. We call the action $(m, A)$-proximal, where $A \subseteq G$ and $m \in \mathbb{N}$, if for any $\epsilon>0$ and any 
finite $F \subseteq X$ there are $g \in A$ and $F_{0} \subseteq X$ such that

$$
\left|F_{0}\right| \leq m \text { and } d\left(g F, F_{0}\right)<\epsilon .
$$

Proposition 4.4. Let $G$ be a Polish group. Assume that for each open neighborhood $U$ of 1 there are a natural number $m_{U}$ and a continuous $\left(m_{U}, U\right)$ proximal action of $G$ on an infinite metric space by uniformly continuous maps. Then $G$ is strongly non-locally compact.

Proof. Let $U$ be an open neighborhood of 1 . We need to find a neighborhood $V$ of 1 such that $U \nsubseteq F_{1} V F_{2}$ for any finite $F_{1}, F_{2} \subseteq G$. Fix a natural number $m_{U}$, an infinite metric space $(X, d)$, and an $\left(m_{U}, U\right)$-proximal action of $G$ on $X$ by uniformly continuous functions as in the assumption of the present proposition. Let $F_{0} \subseteq X$ be an $m_{U}+1$ element set. Continuity of the action of $G$ allows us to pick $\epsilon_{0}>0$ and an open set $1 \in V \subseteq G$ such that

$$
\forall g \in V \forall F \subseteq G\left(|F| \leq m_{U} \Rightarrow d\left(g F_{0}, F\right)>\epsilon_{0}\right) .
$$

We claim that $V$ is as required. Let $g_{i}, h_{i} \in G, i \leq n$. We show that $U \nsubseteq \bigcup_{i \leq n} g_{i} V h_{i}$. By uniform continuity of each $g_{i}^{-1}$, we can find $\delta_{0}>0$ such that for all $i \leq n$ and for any $x, y \in X$

$$
d(x, y)<\delta_{0} \Rightarrow d\left(g_{i}^{-1} x, g_{i}^{-1} y\right)<\epsilon_{0} .
$$

Further, by $\left(m_{U}, U\right)$-proximality of the action, we can find $\bar{g} \in U$ and $\bar{F} \subseteq G$ such that

$$
|\bar{F}| \leq m_{U} \text { and } d\left(\bar{g} \bigcup_{i \leq n} h_{i}^{-1} F_{0}, \bar{F}\right)<\delta_{0} .
$$

Now by (4.20) and (4.21) for any $i \leq n$ there is a subset $\bar{F}_{i}$ of $\bar{F}$ with

$$
d\left(g_{i}^{-1} \bar{g} h_{i}^{-1} F_{0}, g_{i}^{-1} \bar{F}_{i}\right)<\epsilon_{0} .
$$

Since for each $i \leq n,\left|g_{i}^{-1} \bar{F}_{i}\right| \leq m_{U}$, by (4.19), this immediately implies that $g_{i}^{-1} \bar{g} h_{i}^{-1} \notin V$ for all $i \leq n$, that is,

$$
\bar{g} \in U \backslash \bigcup_{i \leq n} g_{i} V h_{i}
$$

and the proposition follows.

4.3.3. Examples. We give here examples of Polish groups which have free subgroups at 1 and are strongly non-locally compact.

1. $\prod_{n} H_{n}$ where $H_{n}, n \in \mathbb{N}$, are Polish groups containing discrete nonAbelian free subgroup.

That this group has a free subgroup at 1 and is strongly non-locally compact follows directly from Propositions 4.1 and 4.3(ii).

2. The group $S_{\infty}$ of all permutations of $\mathbb{N}$ and the group $\operatorname{Aut}(\mathbb{Q})$ of all permutations of $\mathbb{Q}$ which preserve the standard linear order where $\mathbb{Q}$ stands 
for the rationals. Both these groups are taken with the pointwise convergence topology.

First we show that $\operatorname{Aut}(\mathbb{Q})$ has a free subgroup at 1 . Note that $\operatorname{Aut}(\mathbb{Q})$ contains $\operatorname{Aut}(\mathbb{Q})^{\mathbb{N}}$ as a closed subgroup. Indeed, $\operatorname{Aut}(\mathbb{Q})$ is naturally isomorphic, via an isomorphism $\phi_{n}$, with the subgroup of Aut $(\mathbb{Q})$ consisting of all $f$ with $f(q)=q$ for all $q \leq n$ and all $q \geq n+1$ for $n \in \mathbb{N}$. Then the function $\phi: \operatorname{Aut}(\mathbb{Q})^{\mathbb{N}} \rightarrow \operatorname{Aut}(\mathbb{Q})$ given by

$$
\phi\left(\left(f_{n}\right)_{n}\right)=\phi_{0}\left(f_{0}\right) \cdot \phi_{1}\left(f_{1}\right) \cdot \phi_{2}\left(f_{2}\right) \cdots
$$

is well defined (the product converges) and continuous. Moreover, it is a topological isomorphism with the closed subgroup consisting of all $f \in \operatorname{Aut}(\mathbb{Q})$ with $f(n)=n$ for all $n \in \mathbb{N}$ and $f(q)=q$ for all negative $q \in \mathbb{Q}$. Thus, by Proposition 4.1, it suffices to show that $\operatorname{Aut}(\mathbb{Q})$ contains a discrete non-Abelian free subgroup. An explicit example of a non-Abelian free subgroup of $\operatorname{Aut}(\mathbb{Q})$, which one can easily check to be discrete, is given in [3] (see also [14, pp. $30-31]$ ) and another one, with a full argument, in [28, Theorem 8.1].

By identifying $\mathbb{Q}$ with $\mathbb{N}$ via a bijection, we see that $\operatorname{Aut}(\mathbb{Q})$ is a closed subgroup of $S_{\infty}$. Therefore, $S_{\infty}$ has a free subgroup at 1 as well.

Now we show that $\operatorname{Aut}(\mathbb{Q})$ and $S_{\infty}$ are strongly non-locally compact. The proofs in both cases are similar and rely on Proposition 4.4. Consider the natural action of $S_{\infty}$ on the one point compactification $X$ of $\mathbb{N}$ (with some fixed metric): an element of $S_{\infty}$ permutes points of $\mathbb{N}$ and leaves the point at infinity fixed. It is easily seen that if $U$ is an open neighborhood of the identity in $S_{\infty}$ and $n_{U} \in \mathbb{N}$ is such that all permutations $f$ of $\mathbb{N}$ with $f(i)=i$ for all $i \leq n_{U}$ are in $U$, then the action on $X$ is $\left(n_{U}+1, U\right)$-proximal. The action is obviously continuous and, since $X$ is compact, it is done by uniformly continuous functions (with respect to the fixed metric on $X$ ). Thus $S_{\infty}$ is strongly non-locally compact by Proposition 4.4.

The proof that $\operatorname{Aut}(\mathbb{Q})$ is strongly non-locally compact is essentially the same: we consider $\mathbb{Q}$ with the discrete topology and the natural action of $\operatorname{Aut}(\mathbb{Q})$ on the one point compactification of $\mathbb{Q}$ and apply Proposition 4.4 as above.

The example of the group $\operatorname{Aut}(\mathbb{Q})$ is particularly interesting since despite having a free subgroup at 1 this group is extremely amenable, that is, each continuous action of it on a compact space has a fixed point by [28].

3. The group Homeo $\left([0,1]^{n}\right)$ of all homeomorphisms of the $n$ dimensional cube where $n \in \mathbb{N}$.

Fix $n$. Put $G=\operatorname{Homeo}\left([0,1]^{n}\right)$.

Since Homeo( $[0,1])$ is a closed subgroup of $G$, to show that the latter group has a free subgroup at 1 it suffices to see that the former does. In fact, we will show that the group of all increasing homeomorphisms of $[0,1]$ has a free 
subgroup at 1. Using example 2 above, this follows from the fact that $\operatorname{Aut}(\mathbb{Q})$ is a closed subgroup of Homeo([0,1]). In order to see it, consider the $1 / 3$-Cantor subset $C$ of $[0,1]$. Since the order of the family of maximal open intervals included in $[0,1] \backslash C$ induced by the standard ordering of $[0,1]$ is isomorphic to $\mathbb{Q}$ with the standard ordering, it is easy to see that $\operatorname{Aut}(\mathbb{Q})$ is isomorphic as a topological group to the subgroup of $\operatorname{Homeo}([0,1])$ consisting of all increasing homeomorphisms $h:[0,1] \rightarrow[0,1]$ such that $h(C)=C$ and $h\lceil I$ is linear for any interval $I$ included in $[0,1] \backslash C$. This subgroup of $\operatorname{Homeo}([0,1])$ is readily seen to be closed.

To see that $G$ is strongly non-locally compact, we will apply Proposition 4.4. Fix a metric $d$ on $[0,1]^{n}$. Let $D$ be the metric on Homeo $\left([0,1]^{n}\right)$ induced by $d$ via

$$
D(h, f)=\sup _{x \in[0,1]^{n}} d(h(x), f(x)) .
$$

For any open neighborhood $U$ of the identity in $G$ consider the natural action of $G$ on $(0,1)^{n}$. Let $\delta>0$ be such for any homeomorphism $h$ with $D(h, i d)<\delta$ we have $h \in U$. Let $m_{U}$ be $([3 / \delta]+1)^{n}$ and let $\epsilon>0$ be given. Let $F \subseteq(0,1)^{n}$ be a finite set. Divide $(0,1)^{n}$ into $m_{U}$ many cubes using hyperplanes which do not contain points in $F$ and which are parallel to the hyperplanes in $\mathbb{R}^{n}$ given by the equations $x_{i}=0$ for $i=1, \ldots, n$. This is to be done in such a way that each of the cubes in the division has each of its sides of length $<\delta / 2$. Let $B_{k}$, $k \leq m_{U}$, list the interiors of these cubes. So $F \subseteq \bigcup_{k \leq m_{U}} B_{k}$. Let $a_{k} \in B_{k}$. It is easy to see that for each $k$ with $F \cap B_{k} \neq \emptyset$ there exists $h_{k} \in \operatorname{Homeo}\left([0,1]^{n}\right)$ such that $h_{k}$ is the identity on $[0,1]^{n} \backslash B_{k}$ and $h_{k}\left(F \cap B_{k}\right)$ is contained in a ball of radius $\epsilon$ around $a_{k}$. If $F \cap B_{k}=\emptyset$, then let $h_{k}$ be the identity. Then the distance from $h_{k} \circ \cdots \circ h_{1}(F)$ to a subset of $\left\{a_{k}: k \leq m_{U}\right\}$ is not bigger than $\epsilon$ and $D\left(h_{k} \circ \cdots \circ h_{1}, i d\right)<\delta$ since the diameter of each $B_{k}$ is smaller than $\delta$. It follows that $h_{k} \circ \cdots \circ h_{1} \in U$. Thus, we showed that the action of $G$ on $(0,1)^{n}$ is $\left(m_{U}, U\right)$-proximal. Note also that this action is continuous and that it is done by uniformly continuous with respect to $d$ functions as it is a restriction of the natural action of $G$ on the compact metric space $[0,1]^{n}$.

Note that since as shown above the group of all increasing homeomorphisms of $[0,1]$ has a free subgrop at 1 , we see that that Corollary 6(i) generalizes [30].

By an argument similar to the one above one can show that many other homoeomorphism groups of compact metric spaces have free subgroups at 1 and are non-locally compact, for example, Homeo $\left([0,1]^{\mathbb{N}}\right)$ and $\operatorname{Homeo}\left(S^{1}\right)$ where $S^{1}$ is the circle. It may be worth mentioning here that one can deduce from results of [22] (see also [11]) that all non-compact subgroups of the homeomorphism group of $S^{1}$ contain discrete non-Abelian free subgroups. 
4. The group Homeo $\left(2^{\mathbb{N}}\right)$ of all homeomorphisms of $2^{\mathbb{N}}$ and the group of all measure preserving homeomorphisms of $2^{\mathbb{N}}$ where $2^{\mathbb{N}}$ is taken with the product measure $\lambda$ of measures on $2=\{0,1\}$ assigning weight $1 / 2$ to 0 and 1 .

Let us denote the group of measure preserving homeomorphisms of $2^{\mathbb{N}}$ by $G$. An easy argument shows that $G$ contains $G^{\mathbb{N}}$ as a closed subgroup. Simply note that there is a measure preserving homeomorhism between $\left(2^{\mathbb{N}}\right)^{\mathbb{N}}$ with the product measure $\lambda^{\mathbb{N}}$ and $2^{\mathbb{N} \times \mathbb{N}}$ with the product measure of measures on 2 assigning weights $1 / 2$ to both 0 and 1 . By identifying $\mathbb{N}$ with $\mathbb{N} \times \mathbb{N}$ via a bijection we see that this last space is isomorphic to $2^{\mathbb{N}}$ with $\lambda$. Now $G^{\mathbb{N}}$ embeds in the obvious way as a closed subgroup of the group of measure preserving homeomorphisms of $\left(2^{\mathbb{N}}\right)^{\mathbb{N}}$ with $\lambda^{\mathbb{N}}$ so also as a closed subgroup of $G$. Thus, by Proposition 4.1 it suffices to show that $G$ contains a discrete non-Abelian free subgroup. A bijection between $\mathbb{N}$ and the free group $F_{2}$ on two generators induces a measure preserving homeomorphism between $2^{\mathbb{N}}$ and $2^{F_{2}}$. It will be enough to find a discrete non-Abelian free subgroup in the group of measure preserving homeomorphisms of $2^{F_{2}}$. For $\sigma \in F_{2}$ let $S_{\sigma}: 2^{F_{2}} \rightarrow 2^{F_{2}}$ be the shift given by

$$
S_{\sigma}(x)(\tau)=x(\tau \sigma)
$$

Then $\left\{S_{\sigma}: \sigma \in F_{2}\right\}$ is the required discrete subgroup.

Since $G$ is a closed subgroup of $\operatorname{Homeo}\left(2^{\mathbb{N}}\right)$, this last group also has a free subgroup at 1 .

One shows that both groups in this point are strongly non-locally compact by applying Proposition 4.4 to their natural actions on $2^{\mathbb{N}}$. We leave the details to the reader.

5. Given a metric $d$ on $\mathbb{N}^{\mathbb{N}}$ one produces a metric $\rho$ on the free group on $\mathbb{N}^{\mathbb{N}}$ as in [12, pp. $\left.8-10\right]$. This metric is both left and right invariant [12, p. 13]. Let $G$ be the group which is the metric completion with respect to $\rho$ of the free group on $\mathbb{N}^{\mathbb{N}}$. This group is surjectively universal for Polish groups with two-sided invariant metrics, that is, it can be mapped via a continuous homomorphism onto any Polish group with a two-sided invariant metric.

The group $G$ has a free subgroup at 1 . Indeed, pick $x_{n}, x \in \mathbb{N}^{\mathbb{N}}, n \in \mathbb{N}$, all distinct and such that $\lim _{n} d\left(x_{n}, x\right)=0$. Then the group $F$ generated by $\{x\} \cup\left\{x_{n}: n \in \mathbb{N}\right\}$ is free and not discrete. On the other hand, if $D \subseteq$ $\{x\} \cup\left\{x_{n}: n \in \mathbb{N}\right\}$ is finite, then it is easy to verify using formulas defining $\rho$ $[12$, pp. $8-10]$ that for any $g \neq 1$ in the group generated by $D$

$$
\rho(g, 1) \geq \min \left\{d\left(y_{1}, y_{2}\right): y_{1}, y_{2} \in D, y_{1} \neq y_{2}\right\}>0 .
$$

Thus, the group generated by $D$ is discrete. It follows that $F$ witnesses that $G$ has a free subgroup at 1 . 
The group $G$ has a two sided invariant metric and is not locally compact (as it contains a closed copy of $\mathbb{N}^{\mathbb{N}}$ ) so it is strongly non-locally compact by Proposition 4.3(i).

\section{Questions}

One can define the notion of right Haar null sets by changing $\mu(g B)$ in the definition of left Haar null sets to $\mu(B g)$. It follows from Theorem 3(i) that on Polish groups with free subgroup at 1, left Haar null sets are distinct from right Haar null sets. (Simply note that if the set $B$ in the statement of Theorem 3(i) were right Haar null so would be $B \cup B g$ but this last set is equal to $G$.) On the other hand, these two families obviously coincide on Abelian and on locally compact Polish groups and one can check that the same holds on countable products of amenable, locally compact, second countable groups. In this context, the following question is of interest.

Question 1. Let $G$ be an amenable at 1 Polish group. Do left Haar null subsets of $G$ coincide with right Haar null subsets of $G$ ?

It would be interesting to determine more precisely how large the class of amenable at 1 Polish groups is.

Question 2. What other Polish groups except those listed in Proposition 3.2 are among amenable at 1 groups?

An affirmative answer the the next question would allow us to remove the assumption of strong non-local compactness from Theorem 3(ii).

Question 3. Assume $G$ is a Polish group having a free subgroup at 1 . Is $G$ strongly non-locally compact?

In relation to Question 3, one should mention that its "non-compactness" analog is true. It was proved in [31, Lemma 1.2], and independently by Uspenskij as mentioned in [36], that a Polish group $G$ is non-compact precisely when there exists a neighborhood $V$ of 1 such that $G$ is not covered by finitely many sets of the form $g_{1} V g_{2}$ with $g_{1}, g_{2} \in G$.

\section{REFERENCES}

[1] N. Aronszajn, Differentiability of Lipschitzian mappings between Banach spaces, Studia Math. 57(1976), 147-190.

[2] H. Becker, A.S. Kechris, The Descriptive Set Theory of Polish Group Actions, London Mathematical Society Lecture Notes Series 232, Cambridge University Press, 1996.

[3] C.D. Bennett, Explicit free subgroups of Aut $(\mathbb{R}, \leq)$, Proc. Amer. Math. Soc. 125(1997), 1305-1308.

[4] Y. Benyamini, J. Lindenstrauss, Geometric Nonlinear Functional Analysis, American Mathematical Society Colloquium Publications, 48, American Mathematical Society, 2000.

[5] P. Billingsley, Probability and Measure, Wiley, 1979. 
[6] J.P.R. Christensen, Borel structures in groups and semigroups, Math. Scand. 28(1971), 124-128.

[7] J.P.R. Christensen, On sets of Haar measure zero in Abelian Polish groups, Israel J. Math. 13(1972), 255-260.

[8] J.P.R. Christensen, Topology and Borel Structure, North-Holland, 1974.

[9] J. Diestel, Sequences and Series in Banach Spaces, Springer, 1984.

[10] S. Graf, R.D. Mauldin, S.C. Williams, Random homeomorphisms, Adv. in Math. 60 (1986), 239-359.

[11] E. Ghys, Groups acting on the circle, Enseign. Math. 47(2001), 329-407.

[12] M.I. Graev, Free topological groups, American Mathematical Society Translations 35, 1951.

[13] M. Hall, The Theory of Groups, Macmillan, 1959.

[14] P. de la Harpe, Topics in Geometric Group Theory, Chicago Lectures in Mathematics, University of Chicago Press, 2000.

[15] P. de la Harpe, R.I. Grigorchuk, T.G. Ceccherini-Silberstein, Paradoxical decompositions for pseudogroups and discrete metric spaces, Proc. Steklov Inst. Math. 224(1999), 57-97.

[16] E. Hewitt, K.A. Ross, Abstract Harmonic Analysis, Vol. I, Springer, 1963.

[17] G. Hjorth, Classification and Orbit Equivalence Relations, Mathematical Surveys and Monographs, 75, American Mathematical Society, 2000.

[18] B.R. Hunt, T. Saur, J.A. Yorke, Prevalence: a translation invariant "almost every" on infinite dimensional spaces, Bull. Amer. Math. Soc. (N.S.) 27(1992), 217-238.

[19] A.S. Kechris, Classical Descriptive Set Theory, Springer, 1995.

[20] A.S. Kechris, C. Rosendal, Turbulence, amalgamation, and generic automorphisms of homogeneous structures, preprint 2004.

[21] J. Lindenstrauss, D. Preiss, On Frechet differentiability of Lipschitz maps between Banach spaces, Ann. of Math. 157(2003), 257-288.

[22] G. Margulis, Free subgroups of the homeomorphism group of the circle, C. R. Acad. Sci. Paris, 331(2000), 669-674.

[23] E. Matoušková, Translating finite sets into convex sets, Bull. London Math. Soc. 33(2001), 711-714.

[24] J. Mycielski, Some unsolved problems on the prevalence of ergodicity, instability, and algebraic independence, Ulam Quart. 1(1992), 30-37.

[25] O.A. Nielsen, Direct Integral Theory, Lecture Notes in Pure and Applied Mathematics, 61, Marcel Dekker, 1980.

[26] J.C. Oxtoby, Invariant measures in groups which are not locally compact, Trans. Amer. Math. Soc. 60(1946), 215-237.

[27] A.L.T. Paterson, Amenability, Mathematical Surveys and Monographs 29, Amer. Math. Soc., 1988.

[28] V. Pestov, On free actions, minimal flows, and a problem by Ellis, Trans. Amer. Math. Soc. 350(1998), 4149-4165.

[29] V. Pestov, mm-spaces and group actions, Enseign. Math. 48(2002), 209-236.

[30] H. Shi, B.S. Thomson, Haar null sets in the space of automorphisms on [0,1], Real Anal. Exchange 24(1998/99), 337-350.

[31] S. Solecki, Actions of non-compact and non-locally compact Polish groups, J. Symbolic Logic 65(2000), 1881-1894.

[32] S. Solecki, Haar null and non-dominating sets, Fund. Math. 170(2001), 197-217.

[33] S. Solecki, Measuring subsets of groups and Haar null sets, Geom. Funct. Anal., to appear. 
[34] H. Steinhaus, Sur les distances des points dans les ensembles de mesure positive, Fund. Math. 1(1920), 93-104.

[35] F. Topsøe, J. Hoffman-Jørgensen, Analytic spaces and their applications, in Analytic Sets, eds. C.A. Rogers et. al., Academic Press (1980), pp. 317-401.

[36] V. Uspenskij, On subgroups of minimal topological groups, preprint 2000.

[37] A. Weil, L'Integration dans les Groupes Topologiques et ses Applications, Hermann, 1938.

[38] W. Woess, Random Walks on Infinite Graphs and Groups, Cambridge Tracts in Mathematics, 138, Cambridge University Press, 2000.

Department of Mathematics, 1409 W. Green St., University of Illinois, UrBANA, IL 61801

E-mail address: ssolecki@math.uiuc.edu 